Acta Crystallographica Section B

Structural

Science

ISSN 0108-7681

Editor: Carolyn P. Brock

\title{
Neutron Diffraction Study of Aromatic Hydrogen Bonds:
}

5-Ethynyl-5H-dibenzo[a, $d]$ cyclohepten-5-ol at 20K

T. Steiner, S. A. Mason and M. Tamm

This electronic document was scanned from an archival copy of material deposited to accompany a paper published in an IUCr journal. In many cases the only accessible copy was a microfilm of a poor-quality original. 


\section{Supplementary Material to KA035 (Acta Cryst. B)}

First Neutron Diffraction Study of Aromatic Hydrogen Bonds:

\section{5-Ethynyl-5 $H$-dibenzo $[a, d]$ cyclohepten-5-ol at $15 \mathrm{~K}$}

Thomas Steiner, ${ }^{*}, a$ SAX A. Mason ${ }^{b}$ And Matthias TAMM ${ }^{c}$

a Institut für Kristallographie, Freie Universität Berlin, Takustraße 6, D-14195 Berlin, Germany

${ }^{b}$ Institut Laue-Langevin, B. P. 156, 38042 Grenoble Cedex 9, France

c Institut für Anorganische und Analytische Chemie, Freie Universität Berlin, Fabeckstraße 34-36, D-14195 Berlin, Germany

\section{Abstract}

The neutron diffraction crystal structure of the title compound, $\mathrm{C}_{17} \mathrm{H}_{12} \mathrm{O}$, was determined at $20 \mathrm{~K}$. One of the benzyl groups accepts intermolecular hydrogen bonds from a hydroxyl and an ethynyl group, one to each face of the ring. The bond donated by the hydroxyl group points almost linearly at an aromatic C-atom with a H …C separation of $2.339(6) \AA$. The bond donated by ethynyl group points at the aromatic midpoint $M$ with a $\mathrm{H} \cdots \mathrm{M}$ separation of $2.587(5)$ $\AA$. The average of the acetylenic $\mathrm{C}-\mathrm{H}$ bond lengths determined by low temperature neutron diffraction is only $1.062(6) \AA$, appreciably shorter than observed for chemically different $\mathrm{C}-\mathrm{H}$ bonds.
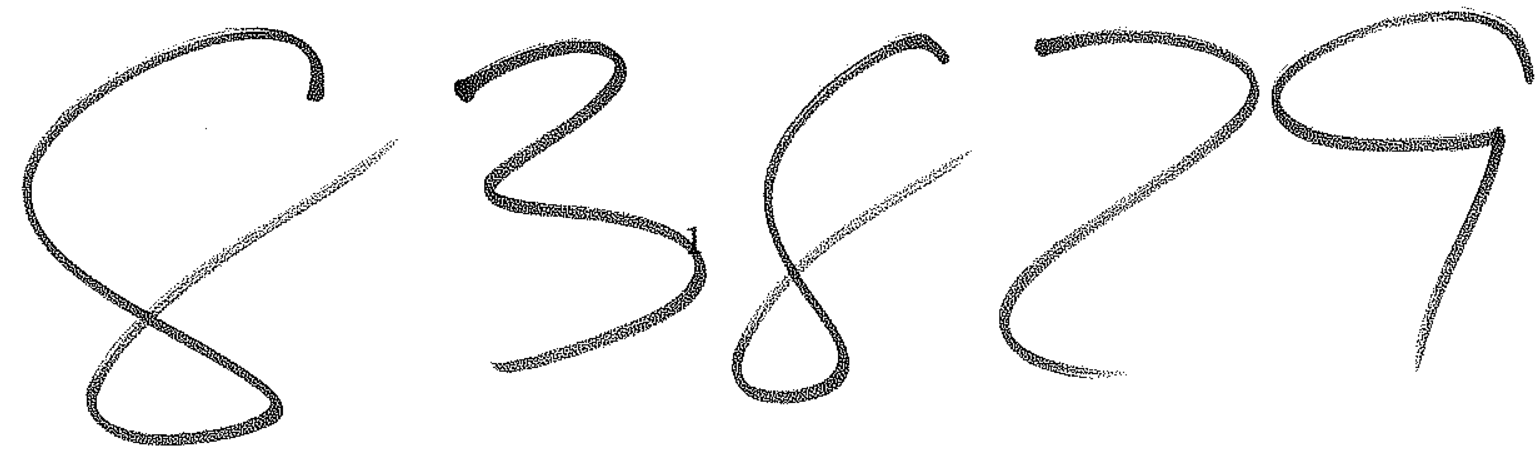
Table 1. Atomic coordinates $\left(x 10^{-4}\right)$ and equivalent isotropic displacement parameters $\left(A^{-2} \times 10^{-3}\right)$ for 1 . U(eq) is defined as one third of the trace of the orthogonalized Uij tensor.

\begin{tabular}{|c|c|c|c|c|}
\hline & $x$ & $y$ & $\mathbf{z}$ & $U(\theta q)$ \\
\hline 0 & $7162(2)$ & $610(3)$ & $3086(2)$ & $9(1)$ \\
\hline$c(1)$ & $9991(2)$ & $3650(2)$ & $1280(2)$ & $10(1)$ \\
\hline$c(2)$ & $9471(2)$ & $5058(2)$ & $1624(2)$ & $10(1)$ \\
\hline$C(3)$ & $8579(2)$ & $4954(3)$ & $2301(2)$ & $11(1)$ \\
\hline$c(4)$ & $8181(2)$ & $3446(2)$ & $2604(2)$ & $9(1)$ \\
\hline$c(5)$ & $8157(2)$ & $381(2)$ & $2531(2)$ & $7(1)$ \\
\hline$c(6)$ & $8676(2)$ & $-1133(2)$ & $4345(2)$ & $10(1)$ \\
\hline$c(7)$ & $9370(2)$ & $-2189(2)$ & $5006(2)$ & $11(1)$ \\
\hline$c(8)$ & $10377(2)$ & $-2752(2)$ & $4634(2)$ & $11(1)$ \\
\hline$c(9)$ & $10699(2)$ & $-2226(2)$ & $3605(2)$ & $9(1)$ \\
\hline$c(10)$ & $10475(2)$ & $-672(2)$ & $1881(2)$ & $8(1)$ \\
\hline$c(11)$ & $10291(2)$ & $717(2)$ & $1310(2)$ & $9(1)$ \\
\hline$c(12)$ & $9625(2)$ & $2110(2)$ & $1625(2)$ & $7(1)$ \\
\hline$c(13)$ & $8679(2)$ & $2029(2)$ & $2258(2)$ & $6(1)$ \\
\hline$C(14)$ & $8988(2)$ & $-631(2)$ & $3300(2)$ & $8(1)$ \\
\hline$c(15)$ & $10026(2)$ & $-1151(2)$ & $2933(2)$ & $8(1)$ \\
\hline$c(16)$ & $7844(2)$ & $-520(2)$ & $1478(2)$ & $8(1)$ \\
\hline$C(17)$ & $7493(2)$ & $-1238(2)$ & $645(2)$ & $9(1)$ \\
\hline $\mathrm{H}(\mathrm{O})$ & $6533(5)$ & $744(7)$ & $2567(5)$ & $32(1)$ \\
\hline $\mathrm{H}(1)$ & $10687(4)$ & $3726(6)$ & $740(4)$ & $27(1)$ \\
\hline $\mathrm{H}(2)$ & $9776(4)$ & $6225(5)$ & $1357(4)$ & $27(1)$ \\
\hline $\mathrm{H}(3)$ & $8186(4)$ & $6054(5)$ & $2585(4)$ & $26(1)$ \\
\hline $\mathrm{H}(4)$ & $7461(4)$ & $3370(5)$ & $3104(4)$ & $22(1)$ \\
\hline $\mathrm{H}(6)$ & $7874(4)$ & $-714(6)$ & $4624(4)$ & $25(1)$ \\
\hline $\mathrm{H}(7)$ & $9091(4)$ & $-2579(6)$ & $5811(4)$ & $29(1)$ \\
\hline $\mathrm{H}(8)$ & $10913(4)$ & $-3596(6)$ & $5138(4)$ & $29(1)$ \\
\hline $\mathrm{H}(9)$ & $11496(4)$ & $-2673(6)$ & $3316(4)$ & $28(1)$ \\
\hline $\mathrm{H}(10)$ & $11091(4)$ & $-1510(5)$ & $1572(4)$ & $24(1)$ \\
\hline $\mathrm{H}(11)$ & $10768(4)$ & $916(5)$ & $586(4)$ & $24(1)$ \\
\hline $\mathrm{H}(17)$ & $7179(5)$ & $-1845(7)$ & $-100(4)$ & $36(1)$ \\
\hline
\end{tabular}


Table 2. Bond lengths [A] and angles [deg] for 1.

\begin{tabular}{|c|c|}
\hline $0-c(5)$ & $1.412(3)$ \\
\hline $0-\mathrm{H}(0)$ & $0.934(8)$ \\
\hline$C(1)-C(2)$ & $1.397(3)$ \\
\hline$c(1)-C(12)$ & $1.419(3)$ \\
\hline$C(1)-B(1)$ & $1.092(5)$ \\
\hline$c(2)-c(3)$ & $1.387(3)$ \\
\hline$C(2)-H(2)$ & $1.088(4)$ \\
\hline$c(3)-C(4)$ & $1.393(3)$ \\
\hline$C(3)-H(3)$ & $1.091(4)$ \\
\hline$C(4)-C(13)$ & $1.392(3)$ \\
\hline$C(4)-H(4)$ & $1.084(4)$ \\
\hline$C(5)-C(16)$ & $1.486(3)$ \\
\hline$C(5)-C(14)$ & $1.536(2)$ \\
\hline$C(5)-C(13)$ & $1.545(2)$ \\
\hline$c(6)-c(7)$ & $1.397(3)$ \\
\hline$C(6)-C(14)$ & $1.400(3)$ \\
\hline$C(6)-H(6)$ & $1.088(5)$ \\
\hline$c(7)-C(8)$ & $1.388(3)$ \\
\hline$C(7)-H(7)$ & $1.096(5)$ \\
\hline$c(8)-C(9)$ & $1.392(3)$ \\
\hline$C(8)-H(8)$ & $1.090(4)$ \\
\hline$c(9)-c(15)$ & $1.401(3)$ \\
\hline$C(9)-H(9)$ & $1.096(4)$ \\
\hline$c(10)-c(11)$ & $1.346(3)$ \\
\hline$c(10)-c(15)$ & $1.466(3)$ \\
\hline$C(10)-H(10)$ & $1.093(4)$ \\
\hline$c(11)-c(12)$ & $1.464(3)$ \\
\hline$C(11)-H(11)$ & $1.088(4)$ \\
\hline$c(12)-c(13)$ & $1.405(3)$ \\
\hline$c(14)-c(15)$ & $1.406(3)$ \\
\hline$c(16)-C(17)$ & $1.206(3)$ \\
\hline$C(17)-H(17)$ & $1.064(5)$ \\
\hline$C(5)-0-\mathrm{H}(0)$ & $110.3(4)$ \\
\hline$C(2)-C(1)-C(12)$ & $120.7(2)$ \\
\hline$C(2)-C(1)-H(1)$ & $120.1(3)$ \\
\hline$C(12)-C(1)-H(1)$ & $119.2(3)$ \\
\hline$c(3)-C(2)-c(1)$ & $119.8(2)$ \\
\hline$C(3)-C(2)-H(2)$ & $121.0(3)$ \\
\hline$C(1)-C(2)-H(2)$ & $119.2(3)$ \\
\hline$C(2)-C(3)-C(4)$ & $120.0(2)$ \\
\hline$C(2)-C(3)-H(3)$ & $119.8(3)$ \\
\hline$C(4)-C(3)-H(3)$ & $120.2(3)$ \\
\hline$c(13)-c(4)-c(3)$ & $121.0(2)$ \\
\hline
\end{tabular}




$\begin{array}{ll}C(13)-C(4)-H(4) & 119.3(3) \\ C(3)-C(4)-H(4) & 119.7(3) \\ O-C(5)-C(16) & 108.2(2) \\ O-C(5)-C(14) & 107.7(2) \\ C(16)-C(5)-C(14) & 109.7(2) \\ O-C(5)-C(13) & 110.2(2) \\ C(16)-C(5)-C(13) & 109.7(2) \\ C(14)-C(5)-C(13) & 111.3(2) \\ C(7)-C(6)-C(14) & 120.5(2) \\ C(7)-C(6)-H(6) & 120.5(3) \\ C(14)-C(6)-H(6) & 119.0(3) \\ C(8)-C(7)-C(6) & 120.3(2) \\ C(8)-C(7)-H(7) & 121.0(3) \\ C(6)-C(7)-H(7) & 118.7(3) \\ C(7)-C(8)-C(9) & 119.2(2) \\ C(7)-C(8)-H(8) & 120.6(3) \\ C(9)-C(8)-H(8) & 120.2(3) \\ C(8)-C(9)-C(15) & 121.7(2) \\ C(8)-C(9)-H(9) & 118.9(3) \\ C(15)-C(9)-H(9) & 119.4(3) \\ C(11)-C(10)-C(15) & 127.9(2) \\ C(11)-C(10)-H(10) & 117.0(3) \\ C(15)-C(10)-H(10) & 114.9(3) \\ C(10)-C(11)-C(12) & 127.5(2) \\ C(10)-C(11)-H(11) & 117.6(3) \\ C(12)-C(11)-H(11) & 114.4(3) \\ C(13)-C(12)-C(1) & 118.5(2) \\ C(13)-C(12)-C(11) & 124.9(2) \\ C(1)-C(12)-C(11) & 116.6(2) \\ C(4)-C(13)-C(12) & 119.8(2) \\ C(4)-C(13)-C(5) & 119.6(2) \\ C(12)-C(13)-C(5) & 120.5(2) \\ C(6)-C(14)-C(15) & 119.6(2) \\ C(6)-C(14)-C(5) & 119.7(2) \\ C(15)-C(14)-C(5) & 120.6(2) \\ C(9)-C(15)-C(14) & 118.7(2) \\ C(9)-C(15)-C(10) & 116.0(2) \\ C(14)-C(15)-C(10) & 125.2(2) \\ C(17)-C(16)-C(5) & 174.3(2) \\ C(16)-C(17)-H(17) & 178.6(4) \\ - & \\ C & \end{array}$


Table 3. Anisotropic displacement parameters $\left(A^{-2} \times 10^{-3}\right)$ for 1. The anisotropic displacement factor exponent takes the form:

-2 pi^2 [ h-2 a*-2 U11 + ..

\begin{tabular}{|c|c|c|c|c|c|c|}
\hline & U11 & $\mathrm{U} 22$ & U33 & U23 & U13 & $\mathrm{U} 12$ \\
\hline 0 & $10(1)$ & $7(1)$ & $10(1)$ & $-2(1)$ & $2(1)$ & $-3(1)$ \\
\hline$c(1)$ & $10(1)$ & $10(1)$ & $9(1)$ & $O(1)$ & $-1(1)$ & $-3(1)$ \\
\hline$C(2)$ & $14(1)$ & $5(1)$ & $9(1)$ & $2(1)$ & $-5(1)$ & $-3(1)$ \\
\hline$c(3)$ & $14(1)$ & $9(1)$ & $10(1)$ & $1(1)$ & $-2(1)$ & $1(1)$ \\
\hline$C(4)$ & $10(1)$ & $9(1)$ & $9(1)$ & $-1(1)$ & $O(1)$ & $O(1)$ \\
\hline$c(5)$ & $7(1)$ & $8(1)$ & $5(1)$ & $1(1)$ & $-2(1)$ & $-2(1)$ \\
\hline$c(6)$ & $11(1)$ & $g(1)$ & $9(1)$ & $-2(1)$ & $-1(1)$ & $1(1)$ \\
\hline$c(7)$ & $17(1)$ & $11(1)$ & $5(1)$ & $2(1)$ & $-2(1)$ & $-2(1)$ \\
\hline$c(8)$ & $14(1)$ & $7(1)$ & $9(1)$ & $3(1)$ & $-6(1)$ & $2(1)$ \\
\hline$c(9)$ & $11(1)$ & $6(1)$ & $10(1)$ & $-2(1)$ & $-2(1)$ & $1(1)$ \\
\hline$c(10)$ & $8(1)$ & $8(1)$ & $10(1)$ & $O(1)$ & $1(1)$ & $2(1)$ \\
\hline$c(11)$ & $9(1)$ & $9(1)$ & $8(1)$ & $O(1)$ & $1(1)$ & $1(1)$ \\
\hline$c(12)$ & $7(1)$ & $\sigma(1)$ & $7(1)$ & $-1(1)$ & $-2(1)$ & $1(1)$ \\
\hline$C(13)$ & $7(1)$ & $5(1)$ & $6(1)$ & $2(1)$ & $-1(1)$ & $-2(1)$ \\
\hline$C(14)$ & $12(1)$ & $5(1)$ & $6(1)$ & $1(1)$ & $-4(1)$ & $-1(1)$ \\
\hline$c(15)$ & $12(1)$ & $6(1)$ & $5(1)$ & $-1(1)$ & $-1(1)$ & $-2(1)$ \\
\hline$c(16)$ & $9(1)$ & $7(1)$ & $8(1)$ & $O(1)$ & $-2(1)$ & $-1(1)$ \\
\hline$c(17)$ & $10(1)$ & $11(1)$ & $5(1)$ & $-3(1)$ & $-3(1)$ & $-2(1)$ \\
\hline $\mathrm{H}(0)$ & $19(2)$ & $47(3)$ & $29(3)$ & $-1(2)$ & $O(2)$ & $7(2)$ \\
\hline $\mathrm{H}(1)$ & $25(2)$ & $28(2)$ & $28(2)$ & $3(2)$ & $7(2)$ & $-6(2)$ \\
\hline $\mathrm{H}(2)$ & $36(2)$ & $15(2)$ & $28(2)$ & $3(2)$ & $3(2)$ & $-8(2)$ \\
\hline $\mathrm{H}(3)$ & $35(2)$ & $11(2)$ & $32(2)$ & $-6(2)$ & $3(2)$ & $1(2)$ \\
\hline H(4) & $26(2)$ & $16(2)$ & $26(2)$ & $-3(2)$ & $12(2)$ & $-1(2)$ \\
\hline $\mathrm{B}(6)$ & $26(2)$ & $27(2)$ & $24(2)$ & $6(2)$ & $9(2)$ & $3(2)$ \\
\hline $\mathrm{H}(7)$ & $36(2)$ & $35(2)$ & $18(2)$ & $7(2)$ & $7(2)$ & $1(2)$ \\
\hline $\mathrm{H}(8)$ & $32(2)$ & $27(2)$ & $27(2)$ & $9(2)$ & $-9(2)$ & $11(2)$ \\
\hline $\mathrm{H}(9)$ & $24(2)$ & $32(2)$ & $28(2)$ & $1(2)$ & $2(2)$ & $13(2)$ \\
\hline $\mathrm{H}(10)$ & $26(2)$ & $22(2)$ & $23(2)$ & $O(2)$ & $6(2)$ & $6(2)$ \\
\hline $\mathrm{H}(11)$ & $30(2)$ & $22(2)$ & $23(2)$ & $3(2)$ & $12(2)$ & $1(2)$ \\
\hline $\mathrm{H}(17)$ & $39(3)$ & $45(3)$ & $21(2)$ & $-15(2)$ & $-13(2)$ & $-9(2)$ \\
\hline
\end{tabular}


Table 4. Torsion angles [deg] for 1.

\begin{tabular}{|c|c|}
\hline$C(12)-C(1)-C(2)-C(3)$ & $-0.6(3)$ \\
\hline$C(1)-C(2)-C(3)-C(4)$ & $-2.2(3)$ \\
\hline$C(2)-C(3)-C(4)-C(13)$ & $1.4(3)$ \\
\hline$c(14)-c(6)-c(7)-c(8)$ & $0.0(3)$ \\
\hline$C(6)-C(7)-C(8)-C(9)$ & $1.3(3)$ \\
\hline$c(7)-c(8)-c(9)-c(15)$ & $-0.5(3)$ \\
\hline$C(15)-C(10)-C(11)-C(12)$ & $2.1(3)$ \\
\hline$C(2)-C(1)-C(12)-C(13)$ & $4.2(3)$ \\
\hline$C(2)-C(1)-C(12)-c(11)$ & $-174.3(2)$ \\
\hline$C(10)-C(11)-C(12)-C(13)$ & $-30.2(3)$ \\
\hline$C(10)-C(11)-C(12)-C(1)$ & $148.2(2)$ \\
\hline$C(3)-C(4)-C(13)-C(12)$ & $2.3(3)$ \\
\hline$C(3)-C(4)-C(13)-C(5)$ & $-175.2(2)$ \\
\hline$c(1)-c(12)-c(13)-c(4)$ & $-5.0(3)$ \\
\hline$C(11)-C(12)-C(13)-C(4)$ & $173.4(2)$ \\
\hline$c(1)-c(12)-C(13)-c(5)$ & $172.5(2)$ \\
\hline$C(11)-C(12)-C(13)-C(5)$ & $-9.1(3)$ \\
\hline $0-c(5)-c(13)-c(4)$ & $2.6(2)$ \\
\hline$c(16)-c(5)-c(13)-C(4)$ & $121.7(2)$ \\
\hline$c(14)-c(5)-c(13)-c(4)$ & $-116.8(2)$ \\
\hline $0-C(5)-C(13)-C(12)$ & $-174.8(2)$ \\
\hline$C(16)-C(5)-C(13)-C(12)$ & $-55.8(2)$ \\
\hline$C(14)-C(5)-C(13)-C(12)$ & $65.7(2)$ \\
\hline$c(7)-c(6)-c(14)-C(15)$ & $-2.2(3)$ \\
\hline$c(7)-C(6)-C(14)-c(5)$ & $173.4(2)$ \\
\hline $0-C(5)-C(14)-C(6)$ & $-0.2(2)$ \\
\hline$c(16)-c(5)-c(14)-c(6)$ & $-117.7(2)$ \\
\hline$c(13)-c(5)-c(14)-c(6)$ & $120.7(2)$ \\
\hline $0-C(5)-C(14)-C(15)$ & $175.4(2)$ \\
\hline$C(16)-C(5)-C(14)-C(15)$ & $57.8(2)$ \\
\hline$C(13)-C(5)-C(14)-C(15)$ & $-63.7(2)$ \\
\hline$C(8)-C(9)-C(15)-C(14)$ & $-1.7(3)$ \\
\hline$c(8)-c(9)-c(15)-c(10)$ & $178.9(2)$ \\
\hline$C(6)-C(14)-C(15)-C(9)$ & $3.0(3)$ \\
\hline$C(5)-C(14)-C(15)-C(9)$ & $-172.6(2)$ \\
\hline$C(6)-C(14)-C(15)-C(10)$ & $-177.6(2)$ \\
\hline$C(5)-C(14)-C(15)-C(10)$ & $6.8(3)$ \\
\hline$C(11)-C(10)-C(15)-C(9)$ & $-151.7(2)$ \\
\hline$C(11)-C(10)-C(15)-C(14)$ & $28.9(3)$ \\
\hline $0-C(5)-C(16)-C(17)$ & $6(2)$ \\
\hline$C(14)-C(5)-C(16)-C(17)$ & $123(2)$ \\
\hline$C(13)-C(5)-C(16)-C(17)$ & $-114(2)$ \\
\hline
\end{tabular}


Table 5. Observed and calculated stxucture factors

\begin{tabular}{|c|c|c|c|c|c|c|c|c|c|c|c|c|c|c|c|c|c|c|c|c|c|c|c|c|c|c|c|c|c|}
\hline h & $\mathbf{x}$ & I. 1 & 10F。 & $10 \mathrm{FC}$ & 108 & h & $\mathbf{k}$ & 11 & $10 F \circ 1$ & $10 \mathrm{Fc}$ & $10 s$ & $\mathrm{~h}$ & $\mathbf{k}$ & 11 & 10F० 1 & $10 \mathrm{Fc}:$ & 105 & $\mathbf{h}$ & $\mathbf{k}$ & 110 & 10Fo 10 & 10FC 1 & $10 \mathrm{~s}$ & h. & $\mathbf{k}$ & ]. 1 & OFO 10 & $10 \mathrm{Fc} 10$ & $10 \mathrm{~s}$ \\
\hline 2 & 0 & 01 & 1030 & 1066 & 16 & 0 & 8 & 0 & 276 & 268 & 15 & -2 & 3 & 1 & 262 & 227 & 38 & -3 & 7 & 1 & 242 & 231 & 11 & -1 & 2 & 2 & 364 & 353 & 17 \\
\hline 4 & 0 & 0 & 844 & 877 & 13 & 1 & 8 & 0 & 82 & 60 & 35 & -1 & 3 & 1 & 255 & 346 & 47 & -2 & 7 & 1 & 239 & 204 & 65 & 0 & 2 & 2 & 1010 & 975 & 16 \\
\hline 6 & 0 & 0 & 560 & 588 & 27 & 2 & 8 & 0 & 70 & 39 & 29 & 0 & 3 & 1 & 398 & 385 & 19 & -1 & 7 & 1 & 158 & 115 & 157 & 1 & 2 & 2 & 307 & 357 & 34 \\
\hline 8 & 0 & 0 & 0 & 16 & 1 & 3 & 8 & 0 & 164 & 155 & 10 & 1 & 3 & 1 & 0 & 43 & 1 & 0 & 7 & 1 & 549 & 523 & 22 & 2 & 2 & 2 & 420 & 350 & 25 \\
\hline 0 & 0 & 0 & 610 & 588 & 22 & 4 & 8 & 0 & 332 & 310 & 29 & 2 & 3 & 1 & 764 & 715 & 24 & 1 & 7 & 11 & 1061 & 1068 & 24 & 3 & 2 & 2 & 319 & 272 & 17 \\
\hline & 0 & 0 & 972 & 967 & 8 & 5 & 8 & 0 & 184 & 200 & 19 & 3 & 3 & 1 & 729 & 721 & 24 & 2 & 7 & 1 & 0 & 319 & -1 & 4 & 2 & 2 & 1112 & 1103 & 9 \\
\hline 1 & 1 & 0 & 892 & 837 & 8 & 6 & 8 & 0 & 368 & 381 & 21 & 4 & 3 & 1 & 219 & 178 & 31 & 3 & 7 & 1 & 347 & 342 & 26 & 5 & 2 & 2 & 0 & 16 & 1 \\
\hline 2 & 1 & 0 & 471 & 485 & 24 & 7 & 8 & 0 & 174 & 176 & 13 & 5 & 3 & 1 & 380 & 367 & 24 & 4 & 7 & 1 & 372 & 343 & 22 & 6 & 2 & 2 & 145 & 66 & 89 \\
\hline 3 & 1 & 0 & 81 & 34 & 80 & 1. & 9 & 0 & 167 & 188 & 16 & 6 & 3 & 1 & 450 & 454 & 29 & 5 & 7 & 1 & 410 & 387 & 13 & 7 & 2 & 2 & 170 & 71 & 80 \\
\hline 4 & 1 & 0 & 378 & 404 & 15 & 2 & 9 & 0 & 149 & 106 & 18 & 7 & 3 & 1 & 337 & 323 & 38 & 6 & 7 & 1 & 309 & 299 & 10 & 8 & 2 & 2 & 0 & 28 & 1 \\
\hline 5 & 1 & 0 & 118 & 91 & 84 & 3 & 9 & 0 & 166 & 180 & 20 & 8 & 3 & 1 & 445 & 413 & 38 & 7 & 7 & 1 & 424 & 411 & 10 & 9 & 2 & 2 & 890 & 893 & 25 \\
\hline 6 & 1 & 0 & 680 & 705 & 50 & 4 & 9 & 0 & 267 & 263 & 13 & 9 & 3 & 1 & 901 & 910 & 19 & 8 & 7 & 1 & 0 & 41 & 1 & 10 & 2 & 2 & 205 & 208 & 13 \\
\hline 7 & 1 & 0 & 0 & 90 & 1 & 5 & 9 & 0 & 102 & 88 & 26 & 10 & 3 & 1 & 634 & 642 & 12 & 9 & 7 & 1 & 203 & 219 & 15 & 11 & 2 & 2 & 410 & 412 & 17 \\
\hline 8 & 1 & 0 & 720 & 730 & 17 & -12 & 0 & 1 & 172 & 158 & 23 & 11 & 3 & 1 & 433 & 449 & 16 & -7 & 8 & 1 & 295 & 297 & 20 & 12 & 2 & 2 & 268 & 235 & 27 \\
\hline 9 & 1 & 0 & 93 & 8 & 92 & -10 & 0 & 1 & 0 & 25 & 1 & 12 & 3 & 1 & 215 & 229 & 15 & -6 & 8 & 1 & 670 & 703 & 14 & 13 & 2 & 2 & 719 & 721 & 13 \\
\hline 10 & 1 & 0 & 264 & 262 & 19 & -8 & 0 & 1 & 310 & 355 & 20 & -12 & 4 & 1 & 338 & 345 & 27 & -5 & 8 & 1 & 617 & 589 & 12 & -13 & 3 & 2 & 348 & 284 & 20 \\
\hline 11 & 1 & 0 & 120 & 136 & 10 & -6 & 0 & 1 & 10851 & 1115 & 22 & -11 & 4 & 1 & 332 & 324 & 11 & -4 & 8 & 1 & 248 & 259 & 20 & -12 & 3 & 2 & 87 & 40 & 39 \\
\hline 12 & 1 & 0 & 215 & 228 & 9 & -4 & 0 & 1 & 12751 & 1345 & 17 & -10 & 4 & 1 & 260 & 248 & 7 & -3 & 8 & 1 & 513 & 484 & 9 & -11 & 3 & 2 & 707 & 722 & 20 \\
\hline 13 & 1 & 0 & 415 & 429 & 9 & -2 & 0 & 11 & 17971 & 1937 & 3 & -9 & 4 & 1 & 948 & 946 & 12 & -2 & 8 & 1 & 404 & 398 & 13 & -10 & 3 & 2 & 732 & 718 & 8 \\
\hline 0 & 2 & 0 & 677 & 662 & 17 & 2 & 0 & 1 & 992 & 971 & 13 & -8 & 4 & 1 & 528 & 570 & 18 & -1 & 8 & 1 & 327 & 330 & 17 & -9 & 3 & 2 & 354 & 365 & 11 \\
\hline 1 & 2 & 0 & 458 & 438 & 15 & 4 & 0 & 1 & 0 & 57 & 1 & -7 & 4 & 1 & 282 & 312 & 45 & 0 & 8 & 1 & 76 & 100 & 76 & -8 & 3 & 2 & 119 & 140 & 79 \\
\hline 2 & 2 & 0 & 702 & 702 & 13 & 6 & 0 & 1 & 582 & 669 & 28 & -6 & 4 & 1 & 100 & 79 & 100 & 1 & 8 & 1 & 365 & 362 & 12 & -7 & 3 & 2 & 951 & 986 & 12 \\
\hline 3 & 2 & 0 & 218 & 193 & 27 & 8 & 0 & 1 & 640 & 632 & 26 & -5 & 4 & 1 & 139 & 161 & 129 & 2 & 8 & 1 & 198 & 209 & 14 & -6 & 3 & 2 & 1063 & 1096 & 13 \\
\hline 4 & 2 & 0 & 87 & 53 & 87 & 10 & 0 & 1 & 88 & 107 & 87 & -4 & 4 & 1 & 834 & 629 & 23 & 3 & 8 & 1 & 443 & 453 & 12 & -5 & 3 & 2 & 300 & 307 & 20 \\
\hline 5 & 2 & 0 & 342 & 325 & 24 & 12 & 0 & 1 & 88 & 140 & 55 & -3 & 4 & 1 & 344 & 392 & 30 & 4 & 8 & 1 & 303 & 282 & 16 & -4 & 3 & 2 & 137 & 31 & 137 \\
\hline 6 & 2 & 0 & 0 & 8 & 1 & -13 & 1 & 1 & 572 & 554 & 15 & -2 & 4 & 1 & 368 & 360 & 35 & 5 & 8 & 1 & 34 & 39 & 33 & -3 & 3 & 2 & 1053 & 1082 & 21 \\
\hline 7 & 2 & 0 & 55 & 8 & 55 & -12 & 1 & 1 & 789 & 752 & 11 & -1 & 4 & 1 & 838 & 784 & 11 & 6 & 8 & 1 & 132 & 45 & 30 & -2 & 3 & 2 & 302 & 309 & 25 \\
\hline 8 & 2 & 0 & 288 & 290 & 16 & -11 & 1 & 11 & 1016 & 1015 & 27 & 0 & 4 & 1 & 111 & 30 & 83 & 7 & 8 & 1 & 196 & 184 & 20 & -1 & 3 & 2 & 423 & 392 & 10 \\
\hline 9 & 2 & 0 & 338 & 357 & 14 & -10 & 1 & 1 & 0 & 31 & 1 & 1 & 4 & 1 & 220 & 167 & 33 & -5 & 9 & 1 & 76 & 32 & 76 & 0 & 3 & 2 & 489 & 438 & 17 \\
\hline 10 & 2 & 0 & 369 & 380 & 7 & -9 & 1 & 1 & 553 & 625 & 26 & 2 & 4 & 1 & 428 & 458 & 26 & -4 & 9 & 1 & 321 & 340 & 14 & 1 & 3 & 2 & 247 & 278 & 201 \\
\hline 11 & 2 & 0 & 31 & 71 & 30 & -8 & 1 & 1 & 151 & 184 & 56 & 3 & 4 & 1 & 394 & 447 & 21 & -3 & 9 & 1 & 230 & 207 & 37 & 2 & 3 & 2 & 576 & 545 & 30 \\
\hline 12 & 2 & 0 & 129 & 127 & 1.4 & -7 & 1 & 1 & 271 & 312 & 37 & 4 & 4 & 1 & 812 & 801 & 21 & -2 & 9 & 1 & 208 & 175 & 19 & 3 & 3 & 2 & 191 & 186 & 28 \\
\hline 13 & 2 & 0 & 580 & 608 & 7 & -8 & 1 & 1 & 552 & 626 & 27 & 5 & 4 & 1 & 866 & 835 & 20 & -1 & 9 & 1 & 85 & 5 & 85 & 4 & 3 & 2 & 683 & 651 & 19 \\
\hline 1 & 3 & 0 & 793 & 722 & 20 & -5 & 1 & 1 & 836 & 848 & 20 & 6 & 4 & 1 & 521 & 516 & 29 & 0 & 9 & 1 & 150 & 77 & 27 & 5 & 3 & 2 & 495 & 523 & 18 \\
\hline 2 & 3 & 0 & 379 & 367 & 23 & -4 & 1 & 1 & 73 & 41 & 72 & 7 & 4 & 1 & 814 & 850 & 25 & 1 & 9 & 1 & 869 & 872 & 12 & 6 & 3 & 2 & 840 & 862 & 22 \\
\hline 3 & 3 & 0 & 111 & 86 & 110 & -3 & 1 & 1 & 757 & 784 & 19 & 8 & 4 & 1 & 611 & 596 & 30 & 2 & 9 & 1 & 115 & 67 & 36 & 7 & 3 & 2 & 531 & 523 & 26 \\
\hline 4 & 3 & 0 & 615 & 586 & 16 & -2 & 1 & 1 & 112 & 132 & 66 & 9 & 4 & 1 & 468 & 450 & 12 & 3 & 9 & 1 & 284 & 269 & 13 & 8 & 3 & 2 & 1883 & 1862 & 21 \\
\hline 5 & 3 & 0 & 641 & 646 & 14 & -1 & 1 & 1 & 835 & 804 & 10 & 10 & 4 & 1 & 523 & 502 & 13 & 4 & 9 & 1 & 115 & 38 & 21 & 9 & 3 & 2 & 204 & 166 & 30 \\
\hline 6 & 3 & 0 & 216 & 186 & 43 & 0 & 1 & 1 & 335 & 292 & 17 & 11 & 4 & 1 & 1233 & 1229 & 11 & -12 & 0 & 2 & 1038 & 1006 & 10 & 10 & 3 & 2 & 292 & 292 & 11 \\
\hline 7 & 3 & 0 & 74 & 27 & 73 & 1 & 1 & 1 & 220 & 244 & 33 & 12 & 4 & 1 & 501 & 525 & 16 & -10 & 0 & 2 & 684 & 747 & 23 & 11 & 3 & 2 & 689 & 686 & 12 \\
\hline 8 & 3 & 0 & 378 & 411 & 16 & 2 & 1 & 1 & 393 & 410 & 23 & -11 & 5 & 1 & 398 & 387 & 13 & -8 & 0 & 2 & 949 & 973 & 21 & 12 & 3 & 2 & 795 & 803 & 14 \\
\hline 9 & 3 & 0 & 296 & 270 & 27 & 3 & 1 & 1 & 706 & 679 & 19 & -10 & 5 & 1 & 462 & 479 & 7 & -6 & 0 & 2 & 1069 & 1094 & 26 & -12 & 4 & 2 & 544 & 517 & 8 \\
\hline 10 & 3 & 0 & 1117 & 1137 & 14 & 4 & 1 & 1 & 442 & 466 & 17 & -9 & 5 & 1 & 84 & 45 & 28 & -4 & 0 & 2 & 87 & 62 & 86 & -11 & 4 & 2 & 230 & 241 & 13 \\
\hline 11 & 3 & 0 & 1106 & 1083 & 6 & 5 & 1 & 1 & 660 & 692 & 17 & -8 & 5 & 1 & 299 & 291 & 11 & -2 & 0 & 2 & 319 & 299 & 23 & -10 & 4 & 2 & 150 & 117 & 16 \\
\hline 12 & 3 & 0 & 192 & 212 & 8 & 6 & 1 & 1 & 1271 & 1295 & 39 & -7 & 5 & 1 & 256 & 243 & 53 & 0 & 0 & 2 & 1412 & 1437 & 9 & -9 & 4 & 2 & 861 & 850 & 6 \\
\hline 13 & 3 & 0 & 437 & 433 & 11 & 7 & 1 & 1 & 1181 & \pm 182 & 13 & -6 & 5 & 1 & 0 & 74 & 1 & 2 & 0 & 2 & 238 & 221 & 17 & -8 & 4 & 2 & 1026 & 1039 & 13 \\
\hline 0 & 4 & 0 & 897 & 921 & 24 & 8 & 1 & 1 & 145 & 159 & 144 & -5 & 5 & 1 & 139 & 97 & 75 & 4 & 0 & 2 & 293 & 301 & 21 & -7 & 4 & 2 & 142 & 102 & 71 \\
\hline 1 & 4 & 0 & 261 & 276 & 48 & 9 & 1 & 1 & 606 & 625 & 14 & -4 & 5 & 1 & 210 & 189 & 47 & B & 0 & 2 & 515 & 557 & 23 & -6 & 4 & 2 & 527 & 543 & 27 \\
\hline 2 & 4 & 0 & 466 & 468 & 19 & 10 & 1 & 1 & 311 & 296 & 30 & -3 & 5 & 1 & 134 & 93 & 133 & 8 & 0 & 2 & 219 & 56 & 56 & -5 & 4 & 2 & 147 & 103 & 93 \\
\hline 3 & 4 & 0 & 263 & 242 & 37 & 11 & 1 & 1 & 134 & 144 & 20 & -2 & 5 & \pm & 723 & 717 & 23 & 10 & 0 & 2 & 1076 & 1035 & 25 & -4 & 4 & 2 & 101 & 106 & 100 \\
\hline & & 0 & 454 & 469 & 11 & 12 & 1 & 1 & $2 \pm 7$ & 217 & 10 & -1 & 5 & 1 & 249 & 300 & 47 & 12 & 0 & 2 & 415 & 377 & 13 & -3 & 4 & 2 & 826 & 819 & 20 \\
\hline
\end{tabular}




\begin{tabular}{|c|c|c|c|c|c|c|c|c|c|c|c|c|c|c|c|c|c|c|c|c|c|c|c|c|c|c|c|c|c|}
\hline 5 & 4 & 0 & 263 & 276 & 17 & 13 & 1 & 1 & 33 & 61 & 32 & 0 & 5 & 1 & 403 & 355 & 32 & -13 & 1 & 2 & 49 & 79 & 49 & -2 & 4 & 2 & 425 & 479 & 30 \\
\hline 6 & 4 & 0 & 680 & 712 & 19 & -13 & 2 & 1 & 879 & 838 & 6 & 1 & 5 & 1 & 287 & 296 & 38 & -12 & 1 & 2 & 75 & 91 & 75 & -1 & 4 & 2 & 82 & 26 & 82 \\
\hline 7 & 4 & 0 & 664 & 660 & 18 & -12 & 2 & 1 & 233 & 215 & 10 & 2 & 5 & 1 & 328 & 283 & 34 & -11 & 1 & 2 & 392 & 388 & 14 & 0 & 4 & 2 & 313 & 253 & 26 \\
\hline 8 & 4 & 01 & 1372 & 1352 & 16 & -11 & 2 & 1 & 484 & 467 & 14 & 3 & 5 & 1 & 262 & 205 & 44 & -10 & 1 & 2 & 388 & 373 & 36 & 1 & 4 & 2 & 219 & 196 & 60 \\
\hline 9 & 4 & 0 & 865 & 857 & 7 & -10 & 2 & 1 & 163 & 116 & 22 & 4 & 5 & 1 & 515 & 549 & 57 & -9 & 1 & 2 & 323 & 307 & 81 & 2 & 4 & 21 & 1184 & 1136 & 11 \\
\hline 10 & 4 & 0 & 755 & 741 & 8 & -9 & 2 & 1 & 425 & 506 & 63 & 5 & 5 & 1 & 21.4 & 239 & 64 & -8 & 1 & 2 & 130 & 85 & 130 & 3 & 4 & 2 & 390 & 415 & 30 \\
\hline 11 & 4 & 0 & 261 & 296 & 11 & -8 & 2 & 1 & 364 & 350 & 14 & 6 & 5 & 1 & 535 & 528 & 22 & -7 & 1 & 2 & 353 & 350 & 19 & 4 & 4 & 2 & 905 & 808 & -11 \\
\hline 2 & 4 & 0 & 815 & 800 & 9 & -7 & 2 & 1 & 286 & 299 & 29 & 7 & 5 & 1 & 873 & 845 & 28 & -8 & 1 & 2 & 151 & 116 & 44 & 5 & 4 & 2 & 205 & 114 & 40 \\
\hline 1 & 5 & 0 & 487 & 494 & 15 & -6 & 2 & 1 & 0 & 112 & 1 & 8 & 5 & 1 & 360 & 352 & 15 & -5 & 1 & 2 & 223 & 280 & 22 & 6 & 4 & 2 & 562 & 556 & 28 \\
\hline 2 & 5 & 0 & 174 & 176 & 51 & -5 & 2 & 11 & 12321 & 1238 & 11 & 9 & 5 & 1 & 905 & 894 & 11 & -4 & 1 & 2 & 334 & 342 & 19 & 7 & 4 & 2 & 728 & 753 & 26 \\
\hline 3 & 5 & 0 & 261 & 216 & 33 & -4 & 2 & 1 & 528 & 563 & 27 & 10 & 5 & 1 & 74 & 101 & 73 & -3 & 1 & 2 & 538 & 574 & 16 & 8 & 4 & 2 & 0 & 12 & 1 \\
\hline 4 & 5 & 0 & 432 & 462 & 20 & -3 & 2 & 1 & 419 & 403 & 18 & 11 & 5 & 1 & 445 & 436 & 16 & -2 & 1 & 2 & 527 & 496 & 20 & 9 & 4 & 2 & 170 & 142 & 15 \\
\hline 5 & 5 & 0 & 217 & 226 & 30 & -2 & 2 & 1 & 0 & 68 & \pm & -10 & 6 & 1 & 267 & 247 & 15 & -1 & $\mathbf{i}$ & 2 & 115 & 178 & 60 & 10 & 4 & 2 & 964 & 932 & 12 \\
\hline 6 & 5 & 0 & 319 & 320 & 16 & -1 & 2 & 1 & 912 & 879 & 17 & -9 & 6 & 1 & 186 & 149 & 17 & 0 & 1 & 21 & 1079 & 1033 & 10 & 11 & 4 & 2 & 586 & 573 & 11 \\
\hline 7 & 5 & 0 & 734 & 707 & 18 & 0 & 2 & 1 & 659 & 669 & 18 & -8 & 6 & 1 & 515 & 510 & 14 & 1 & 1 & 2 & 359 & 338 & 18 & 12 & 4 & 2 & 92 & 41 & 38 \\
\hline 8 & 5 & 0 & 330 & 353 & 11 & 1 & 2 & 1 & 380 & 340 & 23 & -7 & 6 & 11 & 11971 & 1171 & 10 & 2 & 1 & 21 & 1958 & 1843 & 11 & -11 & 5 & 2 & 80 & 34 & 29 \\
\hline 9 & 5 & 0 & 187 & 142 & 35 & 2 & 2 & 1 & 576 & 560 & 17 & -6 & 6 & 11 & 1024 & 942 & 9 & 3 & 1 & 21 & 1003 & 970 & 8 & -10 & 5 & 2 & 108 & 68 & 19 \\
\hline 10 & 5 & 0 & 286 & 258 & 12 & 3 & 2 & 1 & 659 & 645 & 14 & -5 & 6 & 11 & 16521 & 1639 & 12 & 4 & 1 & 2 & 140 & 182 & 108 & -9 & 5 & 2 & 201 & 199 & 10 \\
\hline 11 & 5 & 0 & 136 & 102 & 32 & 4 & 2 & 1 & 509 & 472 & 31 & -4 & 6 & 1 & 208 & 212 & 49 & 5 & 1 & 2 & 635 & 636 & 25 & -8 & 5 & 2 & 806 & 788 & 13 \\
\hline 0 & 6 & 0 & 390 & 337 & 22 & 5 & 2 & 1 & 259 & 205 & 45 & -3 & 6 & 1 & 723 & 707 & 25 & 6 & 1 & 2 & 240 & 180 & 30 & -7 & 5 & 2 & 384 & 406 & 44 \\
\hline 1 & 6 & 0 & 980 & 979 & 22 & 6 & 2 & 1 & 298 & 279 & 32 & -2 & 6 & 1 & 163 & 189 & 94 & 7 & 1 & 2 & 213 & 320 & 56 & -6 & 5 & 2 & 285 & 318 & 26 \\
\hline 2 & 6 & 0 & 478 & 466 & 23 & 7 & 2 & 1 & 179 & 140 & 44 & -1 & 6 & 1 & 845 & 854 & 23 & 8 & 1 & 2 & 569 & 603 & 23 & -5 & 5 & 2 & 334 & 383 & 37 \\
\hline 3 & 6 & 0 & 77 & 78 & 77 & 8 & 2 & 1 & 897 & 931 & 18 & 0 & 6 & 1 & 299 & 329 & 26 & 9 & 1 & 2 & 278 & 243 & 26 & -4 & 5 & 2 & 717 & 712 & 25 \\
\hline 4 & 8 & 0 & 640 & 646 & 17 & 9 & 2 & 1 & 451 & 464 & 19 & 1 & 6 & 1 & 497 & 501 & 20 & 10 & 1 & 2 & 0 & 44 & 1 & -3 & 5 & 2 & 414 & 493 & 19 \\
\hline 5 & 6 & 0 & 217 & 140 & 37 & 10 & 2 & 11 & 10301 & 1004 & 12 & 2 & 6 & 11 & 10191 & 1014 & 15 & 11 & 1 & 2 & 563 & 561 & 12 & -2 & 5 & 2 & 627 & 614 & 26 \\
\hline 6 & 6 & 0 & 750 & 771 & 11 & 11 & 2 & 1 & 531 & 559 & 14 & 3 & 6 & 1 & 243 & 184 & 55 & 12 & 1 & 2 & 225 & 205 & 23 & -1 & 5 & 2 & 108 & 171 & 108 \\
\hline 7 & 6 & 0 & 298 & 284 & 11 & 12 & 2 & 1 & 633 & 606 & 12 & 4 & 6 & 1 & 76 & 101 & 76 & 13 & 1 & 2 & 372 & 373 & 17 & 0 & 5 & 2 & 336 & 322 & 25 \\
\hline 8 & 6 & 0 & 248 & 259 & 9 & 13 & 2 & 1 & 0 & 38 & 1 & 5 & 6 & 1 & 0 & 59 & 1 & -13 & 2 & 2 & 133 & 164 & 31 & 1 & 5 & 2 & 498 & 488 & 18 \\
\hline 9 & 6 & 0 & 442 & 431 & 16 & -13 & 3 & 1 & 119 & 57 & 17 & 6 & 6 & 1 & 566 & 508 & 32 & -12 & 2 & 2 & 693 & 679 & 9 & 2 & 5 & 2 & 0 & 87 & 1 \\
\hline 10 & 6 & 0 & 144 & 138 & 18 & -12 & 3 & 1 & 228 & 195 & 11 & 7 & 6 & 1 & 989 & 985 & 11 & -11 & 2 & 2 & 0 & 5 & 1 & 3 & 5 & 2 & 841 & 666 & 59 \\
\hline 1 & 7 & 01 & 16321 & 1653 & 10 & -11 & 3 & 1 & 159 & 153 & 12 & 8 & 8 & 1 & 438 & 461 & 13 & -10 & 2 & 2 & 333 & 322 & 60 & 4 & 5 & 2 & 33 & 117 & 33 \\
\hline 2 & 7 & 0 & 125 & 53 & 125 & -10 & 3 & 1 & 343 & 348 & 12 & 9 & 6 & 1 & 370 & 383 & 15 & -9 & 2 & 2 & 871 & 840 & 24 & 5 & 5 & 2 & 141 & 1431 & 140 \\
\hline 3 & 7 & 0 & 0 & 46 & 1 & -9 & 3 & 11 & 16181 & 1656 & 8 & 10 & 6 & 1 & 107 & 92 & 39 & -8 & 2 & 2 & 965 & 989 & 20 & 6 & 5 & 2 & 562 & 592 & 34 \\
\hline 4 & 7 & 0 & 535 & 522 & 8 & -8 & 3 & 1 & 759 & 788 & 15 & -9 & 7 & 1 & 497 & 488 & 14 & -7 & 2 & 2 & 233 & 217 & 46 & 7 & 5 & 2 & 138 & 122 & 138 \\
\hline 5 & 7 & 0 & 181 & 177 & 16 & -7 & 3 & 1 & 201 & 145 & 32 & -8 & 7 & 1 & 121 & 87 & 27 & -8 & 2 & 2 & 110 & 159 & 110 & 8 & 5 & 2 & 201 & 158 & 13 \\
\hline 6 & 7 & 0 & 641 & 848 & 16 & -6 & 3 & 1 & 84 & 80 & 83 & -7 & 7 & 1 & 226 & 203 & 13 & -5 & 2 & 2. & 1054 & 1057 & 22 & 9 & 5 & 2 & 0 & 53 & 1 \\
\hline 7 & 7 & 0 & 161 & 132 & 15 & -5 & 3 & 1 & 141 & 92 & 85 & -6 & 7 & 1 & 297 & 303 & 12 & -4 & 2 & 2 & 503 & 534 & 20 & 10 & 5 & 2 & 693 & 679 & 12 \\
\hline 8 & 7 & 0 & 813 & 631 & 7 & -4 & 3 & 1 & 946 & 897 & 23 & -5 & 7 & 11 & 12021 & 1149 & 7 & -3 & 2 & 2 & 403 & 430 & 24 & 11 & 5 & 2 & 626 & 810 & 15 \\
\hline 9 & 7 & 0 & 531 & 561 & 10 & -3 & 3 & 1 & 534 & 509 & 11 & -4 & 7 & 1 & 34 & 3 & 34 & -2 & 2 & 2 & 129 & 63 & 34 & -10 & 6 & 2 & 211 & 235 & 14 \\
\hline-9 & 6 & 2 & 200 & 229 & 12 & 1 & 1 & 3 & 368 & 373 & 28 & -9 & 5 & 3 & 217 & 219 & 14 & 6 & 0 & 4 & 707 & 790 & 24 & -3 & 4 & 4 & 340 & 348 & 36 \\
\hline-8 & 6 & 2 & 235 & 247 & 13 & 2 & 1 & 3 & 391 & 394 & 27 & -8 & 5 & 3 & 29 & 19 & 28 & 8 & 0 & 4 & 95 & 59 & 94 & -2 & 4 & 4 & 58 & 167 & 58 \\
\hline-7 & 6 & 2 & 811 & 796 & 6 & 3 & 1 & 3 & 289 & 249 & 22 & -7 & 5 & 3 & 866 & 884 & 21 & 10 & 0 & 4 & 961 & 933 & 10 & -1 & 4 & 4 & 891 & 887 & 20 \\
\hline-6 & 6 & 2 & 425 & 406 & 9 & 4 & 1 & 3 & 273 & 353 & 43 & -6 & 5 & 3 & 257 & 205 & 56 & 12 & 0 & 4 & 484 & 522 & 16 & 0 & 4 & 4 & 470 & 476 & 19 \\
\hline-5 & 6 & 2 & 0 & 140 & 1 & 5 & 1 & 3 & 824 & 602 & 18 & -5 & 5 & 3 & 242 & 118 & 53 & -13 & 1 & 4 & 42 & 78 & 41 & 1 & 4 & 4 & 52 & 34 & 52 \\
\hline-4 & 6 & 2 & 180 & 11 & 90 & 6 & 1 & 3 & 666 & 653 & 21 & -4 & 5 & 3 & 210 & 271 & 60 & -12 & 1 & 4 & 0 & 17 & 1 & 2 & 4 & 4 & 340 & 369 & 23 \\
\hline-3 & 6 & 2 & 105 & 120 & 81 & 7 & 1 & 3 & 0 & 78 & 1 & -3 & 5 & 3 & 0 & 114 & 1 & -11 & 1 & 4 & 823 & 840 & 11 & 3 & 4 & 4 & 178 & 45 & 75 \\
\hline-2 & 6 & 2 & 367 & 350 & 24 & 8 & 1 & 3 & 895 & 933 & 23 & -2 & 5 & 3 & 747 & 769 & 18 & -10 & 1 & 4 & 375 & 379 & 13 & 4 & 4 & 4 & 810 & 809 & 25 \\
\hline-1 & 6 & 2 & 153 & 101 & 113 & 9 & 1 & 3 & 808 & 771 & 18 & -1 & 5 & 3 & 432 & 505 & 24 & -9 & 1 & 4 & 198 & 178 & 73 & 5 & 4 & 4 & 784 & 816 & 26 \\
\hline 0 & 6 & 2 & 494 & 445 & 31 & 10 & 1 & 31 & 11251 & 1125 & 10 & 0 & 5 & 3 & 0 & 135 & 1 & -8 & 1 & 4 & 144 & 4 & 111 & 6 & 4 & 4 & 47 & 115 & 47 \\
\hline 1 & B & 2 & 869 & 942 & 24 & 11 & 1 & 3 & 224 & 271 & 19 & 1 & 5 & 3 & 21 & 58 & 21 & -7 & 1 & 4 & 244 & 239 & 45 & 7 & 4 & 4 & 554 & 548 & 31 \\
\hline 2 & 6 & 2 & 688 & 665 & 27 & 12 & 1 & 3 & 386 & 397 & 19 & 2 & 5 & 3 & 204 & 237 & 26 & -6 & 1 & 4 & 99 & 36 & 99 & 8 & 4 & 4 & 522 & 499 & 13 \\
\hline 3 & 6 & 21 & 1196 & 1156 & 21 & 13 & 1 & 3 & 81 & 27 & 81 & 3 & 5 & 3 & 234 & 231 & 40 & -5 & 1 & 4 & 712 & 770 & 25 & 9 & 4 & 4 & 262 & 250 & 15 \\
\hline 4 & 6 & 2 & 1111 & 1092 & 19 & -13 & 2 & 3 & 426 & 435 & 16 & 4 & 5 & 3 & 170 & 129 & 85 & -4 & 1 & 4 & 1352 & 1407 & 21 & 10 & 4 & 4 & 323 & 302 & 12 \\
\hline 5 & 6 & 2 & 0 & 137 & 1 & -12 & 2 & 3 & 217 & 225 & 10 & 5 & 5 & 3 & 161 & 25 & 118 & -3 & 1 & 4 & 81 & 79 & 81 & 11 & 4 & 4 & 936 & 967 & 12 \\
\hline 6 & 6 & 2 & 886 & 886 & 11 & $-1 \frac{1}{2}$ & 2 & 3 & 445 & 493 & 15 & 7 & 5 & 3 & $\{41$ & 134 & 27 & -2 & 1 & 4 & 49 & 122 & 49 & -11 & 5 & 4 & 187 & 158 & 20 \\
\hline
\end{tabular}




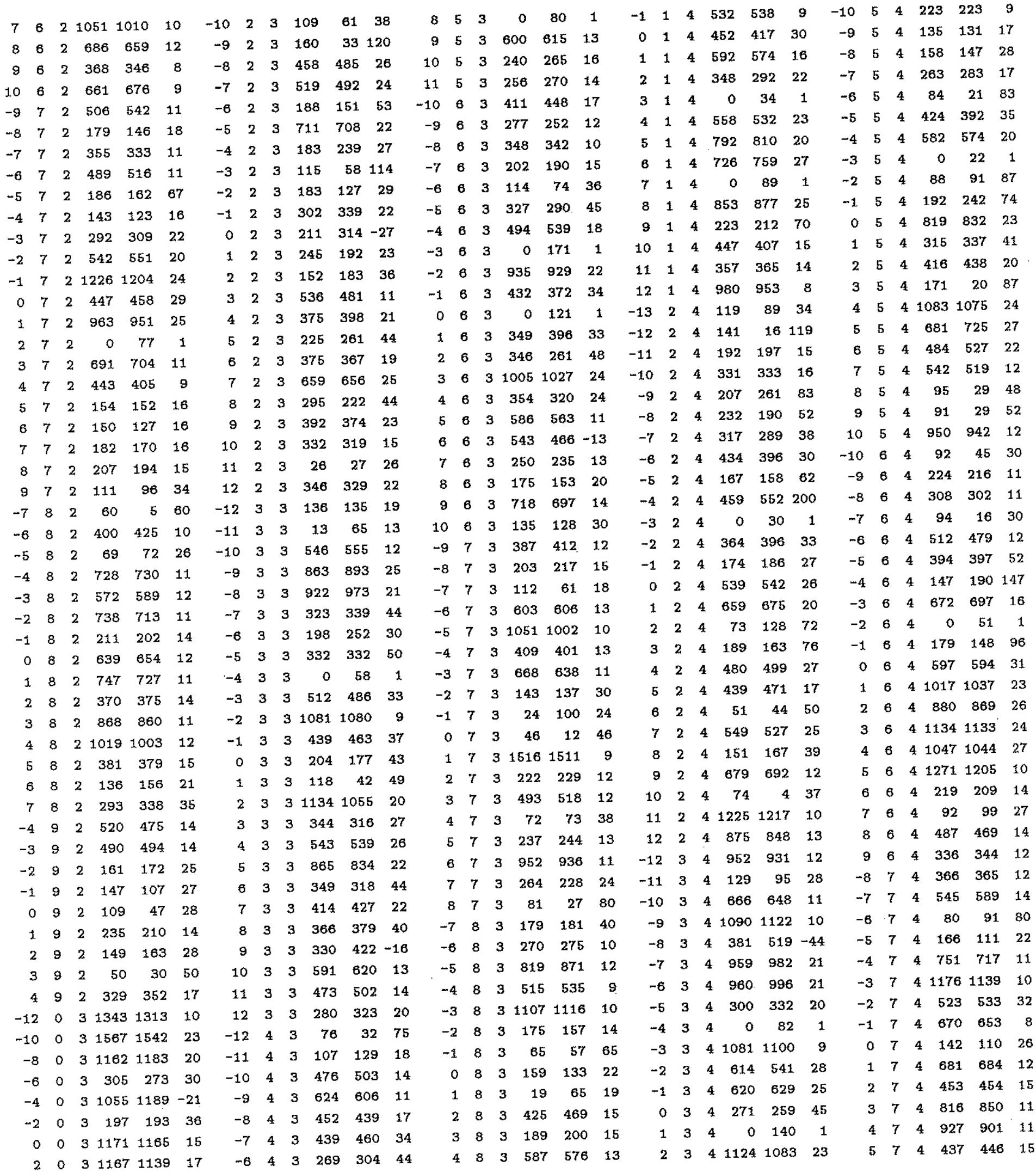


$\begin{array}{llllll}4 & 0 & 3 & 77 & 38 & 76\end{array}$ $\begin{array}{llllll}6 & 0 & 3 & 513 & 523 & 24\end{array}$ $\begin{array}{lllllll}8 & 0 & 3 & 378 & 350 & 31\end{array}$ $10 \quad 0 \quad 3 \quad 1575 \quad 1522 \quad 9$ $\begin{array}{llllll}12 & 0 & 3 & 796 & 816 & 12\end{array}$ $\begin{array}{llllll}-13 & 1 & 3 & 412 & 409 & 12\end{array}$ $\begin{array}{llllll}-12 & 1 & 3 & 123 & 58 & 29\end{array}$ $\begin{array}{llllll}-11 & 1 & 3 & 1069 & 1034 & 10\end{array}$ $\begin{array}{llllll}-10 & 1 & 3 & 409 & 466 & 51\end{array}$ $\begin{array}{llllll}-9 & 1 & 3 & 471 & 518 & 21\end{array}$ $\begin{array}{llllll}-8 & 1 & 3 & 178 & 197 & 84\end{array}$ $\begin{array}{llllll}-7 & 1 & 3 & 773 & 828 & 20\end{array}$ $\begin{array}{llllll}-6 & 1 & 3 & 273 & 234 & 34\end{array}$ $\begin{array}{llllll}-5 & 1 & 3 & 56 & 134 & 56\end{array}$ $\begin{array}{llllll}-4 & 1 & 3 & 933 & 936 & 21\end{array}$ $\begin{array}{llllll}-3 & 1 & 3 & 199 & 210 & 25\end{array}$ $\begin{array}{llllll}-2 & 1 & 3 & 506 & 505 & 24\end{array}$ $\begin{array}{llllll}-1 & 1 & 3 & 167 & 155 & 24\end{array}$

$0 \quad 1 \quad 311041040 \quad 16$ $\begin{array}{llllll}-1 & 9 & 4 & 435 & 460 & 14\end{array}$ $\begin{array}{llllll}0 & 9 & 4 & 184 & 202 & 21\end{array}$ $\begin{array}{llllll}1 & 9 & 4 & 218 & 224 & 14\end{array}$ $\begin{array}{llllll}2 & 9 & 4 & 163 & 153 & 18\end{array}$ $\begin{array}{llllll}-12 & 0 & 5 & 352 & 359 & 15\end{array}$ $\begin{array}{llllll}-10 & 0 & 5 & 513 & 512 & 11\end{array}$ $\begin{array}{llllll}-8 & 0 & 5 & 764 & 731 & 30\end{array}$ $\begin{array}{llllll}-6 & 0 & 5 & 376 & 397 & 33\end{array}$ $\begin{array}{llllll}-4 & 0 & 5 & 853 & 652 & 24\end{array}$ $\begin{array}{lllllll}-2 & 0 & 5 & 688 & 708 & 24\end{array}$ $\begin{array}{llllll}0 & 0 & 5 & 232 & 218 & 27\end{array}$ $\begin{array}{llllll}2 & 0 & 5 & 526 & 503 & 10\end{array}$ $\begin{array}{llllll}4 & 0 & 5 & 1355 & 1312 & 9\end{array}$ $\begin{array}{llllll}6 & 0 & 5 & 710 & 706 & 24\end{array}$ $\begin{array}{lllllll}8 & 0 & 5 & 357 & 409 & 23\end{array}$ 10 $0 \quad 5 \quad 1635 \quad 1661 \quad 10$ $\begin{array}{llllll}12 & 0 & 5 & 699 & 724 & 14\end{array}$ $\begin{array}{llllll}-13 & 1 & 5 & 326 & 297 & 19\end{array}$ $\begin{array}{llllll}-12 & 1 & 5 & 206 & 189 & 10\end{array}$ $\begin{array}{llllll}-11 & 1 & 5 & 145 & 89 & 25\end{array}$ $\begin{array}{llllll}-10 & 1 & 5 & 307 & 290 & 11\end{array}$

$\begin{array}{llllll}-9 & 1 & 5 & 268 & 182 & 53\end{array}$ $\begin{array}{llllll}-8 & 1 & 5 & 653 & 877 & 27\end{array}$ $\begin{array}{llllll}-7 & 1 & 5 & 767 & 790 & 25\end{array}$ $\begin{array}{llllll}-6 & 1 & 5 & 94 & 85 & 93\end{array}$ $\begin{array}{llllll}-5 & 1 & 5 & 188 & 157 & 52\end{array}$ $\begin{array}{llllll}-4 & 1 & 5 & 0 & 100 & 1\end{array}$ $\begin{array}{llllll}-3 & 1 & 5 & 477 & 495 & 27\end{array}$ $\begin{array}{llllll}-2 & 1 & 5 & 288 & 316 & 24\end{array}$ $\begin{array}{llllll}-1 & 1 & 5 & 183 & 199 & 32\end{array}$ $\begin{array}{lllllll}0 & 1 & 5 & 678 & 666 & 25\end{array}$ $\begin{array}{llllll}1 & 1 & 5 & 418 & 417 & 23\end{array}$ $\begin{array}{llllll}2 & 1 & 5 & 1411 & 1322 & 9\end{array}$ $\begin{array}{llllll}3 & 1 & 5 & 0 & 87 & 1\end{array}$ $\begin{array}{llllll}4 & 1 & 5 & 1214 & 1115 & -10\end{array}$ $\begin{array}{llllll}5 & 1 & 5 & 776 & 765 & 21\end{array}$ $\begin{array}{llllll}-5 & 4 & 3 & 361 & 378 & 19\end{array}$ $\begin{array}{llllll}-4 & 4 & 3 & 0 & 34 & 1\end{array}$ $\begin{array}{llllll}-3 & 4 & 3 & 59 & 176 & 58\end{array}$ $\begin{array}{llllll}-2 & 4 & 3 & 257 & 271 & 39\end{array}$ $\begin{array}{llllll}-1 & 4 & 3 & 141 & 17 & 29\end{array}$ - 4 31120101441 $\begin{array}{llllll}1 & 4 & 3 & 201 & 224 & 50\end{array}$ $\begin{array}{llllll}2 & 4 & 3 & 1171 & 1104 & 18\end{array}$ $\begin{array}{llllll}3 & 4 & 3 & 1045 & 1023 & 10\end{array}$ $\begin{array}{llllll}4 & 4 & 3 & 176 & 154 & 72\end{array}$ $\begin{array}{llllll}5 & 4 & 3 & 139 & 161 & 138\end{array}$ $\begin{array}{llllll}6 & 4 & 3 & 360 & 284 & 35\end{array}$ $\begin{array}{llllll}7 & 4 & 3 & 137 & 139 & 137\end{array}$ $\begin{array}{llllll}8 & 4 & 3 & 282 & 237 & 26\end{array}$ $\begin{array}{llllll}9 & 4 & 3 & 84 & 17 & 72\end{array}$ $\begin{array}{llllll}10 & 4 & 3 & 292 & 276 & 12\end{array}$ $\begin{array}{llllll}11 & 4 & 3 & 1088 & 1060 & 12\end{array}$ $\begin{array}{llllll}-11 & 5 & 3 & 762 & 754 & 13\end{array}$ $\begin{array}{llllll}-10 & 5 & 3 & 725 & 726 & 12\end{array}$ $\begin{array}{llllll}10 & 3 & 5 & 0 & 35 & 1\end{array}$ $\begin{array}{llllll}11 & 3 & 5 & 384 & 419 & 18\end{array}$ $\begin{array}{llllll}-11 & 4 & 5 & 195 & 212 & 17\end{array}$ $\begin{array}{llllll}-10 & 4 & 5 & 443 & 458 & 15\end{array}$ $\begin{array}{llllll}-9 & 4 & 5 & 391 & 415 & 14\end{array}$ $\begin{array}{llllll}-8 & 4 & 5 & 1151 & 1138 & 10\end{array}$ $\begin{array}{llllll}-7 & 4 & 5 & 502 & 560 & -28\end{array}$ $\begin{array}{llllll}-6 & 4 & 5 & 1295 & 1312 & 15\end{array}$ $\begin{array}{llllll}-5 & 4 & 5 & 650 & 659 & 26\end{array}$ $\begin{array}{llllll}-4 & 4 & 5 & 757 & 822 & 18\end{array}$ $\begin{array}{llllll}-3 & 4 & 5 & 419 & 428 & 19\end{array}$ $\begin{array}{llllll}-2 & 4 & 5 & 218 & 218 & 39\end{array}$ $\begin{array}{llllll}-1 & 4 & 5 & 438 & 432 & 31\end{array}$ $\begin{array}{llllll}0 & 4 & 5 & 187 & 205 & 67\end{array}$ $\begin{array}{llllll}1 & 4 & 5 & 185 & 214 & 77\end{array}$ 2. $455 \quad 0 \quad 48 \quad 1$ $\begin{array}{llllll}3 & 4 & 5 & 887 & 898 & 16\end{array}$ $\begin{array}{llllll}4 & 4 & 5 & 0 & 104 & 1\end{array}$ $\begin{array}{llllll}5 & 4 & 5 & 374 & 425 & 37\end{array}$ $\begin{array}{llllll}6 & 4 & 5 & 47 & 85 & 47\end{array}$ $\begin{array}{llllll}7 & 4 & 5 & 471 & 466 & 9\end{array}$ $\begin{array}{llllll}8 & 4 & 5 & 87 & 28 & 28\end{array}$ $\begin{array}{llllll}9 & 4 & 5 & 0 & 39 & 1\end{array}$ $\begin{array}{llllll}10 & 4 & 5 & 235 & 240 & 15\end{array}$ $\begin{array}{llllll}11 & 4 & 5 & 327 & 293 & 19\end{array}$ $\begin{array}{llllll}-11 & 5 & 5 & 293 & 355 & 20\end{array}$ $\begin{array}{llllll}-10 & 5 & 5 & 721 & 688 & 14\end{array}$ $\begin{array}{llllll}-9 & 5 & 5 & 766 & 804 & 12\end{array}$ $\begin{array}{llllll}-8 & 5 & 5 & 175 & 189 & 15\end{array}$ $\begin{array}{llllll}-7 & 5 & 5 & 167 & 136 & 23\end{array}$ $\begin{array}{llllll}-6 & 5 & 5 & 0 & 55 & 1\end{array}$ $\begin{array}{llllll}-5 & 5 & 5 & 235 & 107 & 62\end{array}$ $\begin{array}{llllll}-4 & 5 & 5 & 353 & 323 & 38\end{array}$ $\begin{array}{llllll}-3 & 5 & 5 & 0 & 15 & 1\end{array}$ $\begin{array}{llllll}-2 & 5 & 5 & 566 & 540 & 20\end{array}$ $\begin{array}{llllll}-1 & 5 & 5 & 249 & 250 & 53\end{array}$ $\begin{array}{llllll}5 & 8 & 3 & 377 & 374 & 13\end{array}$ $\begin{array}{llllll}6 & 8 & 3 & 143 & 168 & 18\end{array}$ $\begin{array}{llllll}-4 & 9 & 3 & 0 & 22 & 1\end{array}$ $\begin{array}{llllll}-3 & 9 & 3 & 305 & 320 & 12\end{array}$ $\begin{array}{llllll}-2 & 9 & 3 & 728 & 743 & 13\end{array}$ $\begin{array}{llllll}-1 & 9 & 3 & 162 & 183 & 25\end{array}$ $\begin{array}{llllll}0 & 9 & 3 & 396 & 408 & 16\end{array}$ $\begin{array}{llllll}1 & 9 & 3 & 674 & 663 & 13\end{array}$ $\begin{array}{llllll}2 & 9 & 3 & 99 & 81 & 41\end{array}$ $\begin{array}{llllll}3 & 9 & 3 & 187 & 164 & 16\end{array}$ $\begin{array}{lllllll}-12 & 0 & 4 & 345 & 364 & 14\end{array}$ $\begin{array}{llllll}-10 & 0 & 4 & 460 & 467 & 11\end{array}$ $\begin{array}{llllll}-8 & 0 & 4 & 130 & 150 & 129\end{array}$ $\begin{array}{llllll}-6 & 0 & 4 & 0 & 209 & 1\end{array}$ $\begin{array}{lllllll}-4 & 0 & 4 & 1223 & 1268 & 16\end{array}$ $\begin{array}{llllll}-2 & 0 & 4 & 99 & 36 & 56\end{array}$ $\begin{array}{llllll}0 & 0 & 4 & 181 & 155 & 26\end{array}$ $\begin{array}{llllll}2 & 0 & 4 & 489 & 479 & 21\end{array}$ $\begin{array}{llllll}4 & 0 & 4 & 428 & 375 & 22\end{array}$ $\begin{array}{llllll}1 & 8 & 5 & 190 & 186 & 15\end{array}$ $\begin{array}{llllll}2 & 8 & 5 & 193 & 197 & 15\end{array}$ $\begin{array}{llllll}3 & 8 & 5 & 494 & 482 & 16\end{array}$ $\begin{array}{llllll}4 & 8 & 5 & 684 & 663 & 14\end{array}$ $\begin{array}{llllll}5 & 8 & 5 & 948 & 939 & 8\end{array}$ $\begin{array}{llllll}-1 & 9 & 5 & 1175 & 1214 & 12\end{array}$ $\begin{array}{llllll}0 & 9 & 5 & 129 & 90 & 30\end{array}$ $\begin{array}{llllll}-12 & 0 & 6 & 707 & 631 & 14\end{array}$ $\begin{array}{llllll}-10 & 0 & 6 & 766 & 765 & 12\end{array}$ $\begin{array}{lllllll}-8 & 0 & 6 & 1570 & 1564 & 21\end{array}$ $\begin{array}{llllll}-6 & 0 & 6 & 1089 & 1064 & 20\end{array}$ $\begin{array}{llllll}-4 & 0 & 6 & 404 & 454 & 19\end{array}$ $\begin{array}{llllll}-2 & 0 & 6 & 1323 & 1356 & 9\end{array}$ $\begin{array}{llllll}0 & 0 & 6 & 535 & 508 & 39\end{array}$ $\begin{array}{lllllll}2 & 0 & 6 & 1729 & 1712 & 19\end{array}$ $\begin{array}{llllll}4 & 0 & 6 & 656 & 675 & 24\end{array}$ $\begin{array}{llllll}6 & 0 & 6 & 776 & 782 & 13\end{array}$ $\begin{array}{llllll}8 & 0 & 6 & 710 & 721 & 27\end{array}$ $\begin{array}{llllll}10 & 0 & 6 & 511 & 495 & 15\end{array}$ $\begin{array}{llllll}-12 & 1 & 6 & 354 & 360 & 18\end{array}$ $\begin{array}{llllll}-11 & 1 & 6 & 54 & 20 & 54\end{array}$ $\begin{array}{llllll}-10 & 1 & 6 & 343 & 329 & 15\end{array}$ $\begin{array}{llllll}-9 & 1 & 6 & 229 & 227 & 31\end{array}$ $\begin{array}{llllll}-8 & 1 & 6 & 983 & 1043 & 24\end{array}$ $\begin{array}{llllll}-7 & 1 & 6 & 206 & 200 & 33\end{array}$ $\begin{array}{llllll}-6 & 1 & 6 & 381 & 350 & 31\end{array}$ $\begin{array}{llllll}-5 & 1 & 6 & 341 & 338 & 19\end{array}$ $\begin{array}{llllll}-4 & 1 & 6 & 0 & 38 & 1\end{array}$ $\begin{array}{llllll}-3 & 1 & 6 & 357 & 372 & 27\end{array}$ $\begin{array}{llllll}-2 & 1 & 6 & 997 & 1014 & 22\end{array}$ $\begin{array}{llllll}-1 & 1 & 6 & 480 & 471 & 34\end{array}$ $\begin{array}{llllll}0 & 1 & 6 & 304 & 296 & 26\end{array}$ $\begin{array}{llllll}1 & 1 & 6 & 141 & 172 & 42\end{array}$ $\begin{array}{llllll}2 & 1 & 6 & 852 & 866 & 17\end{array}$ $\begin{array}{llllll}3 & 1 & 6 & 143 & 133 & 49\end{array}$ $\begin{array}{llllll}4 & 1 & 6 & 894 & 819 & 22\end{array}$ $\begin{array}{llllll}3 & 3 & 4 & 1386 & 1296 & 18\end{array}$

$\begin{array}{llllll}4 & 3 & 4 & 124 & 110 & 40\end{array}$

$\begin{array}{llllll}5 & 3 & 4 & 51 & 137 & 51\end{array}$

$\begin{array}{llllll}6 & 3 & 4 & 530 & 587 & 31\end{array}$

$\begin{array}{llllll}7 & 3 & 4 & 201 & 81 & 70\end{array}$

$\begin{array}{llllll}8 & 3 & 4 & 273 & 248 & 19\end{array}$

$\begin{array}{llllll}9 & 3 & 4 & 827 & 813 & 11\end{array}$

$\begin{array}{lllllll}10 & 3 & 4 & 57 & 87 & 56\end{array}$

$\begin{array}{llllll}11 & 3 & 4 & 647 & 551 & -14\end{array}$

$\begin{array}{llllll}12 & 3 & 4 & 1048 & 1036 & 12\end{array}$

$\begin{array}{llllll}-12 & 4 & 4 & 134 & 137 & 29\end{array}$

$\begin{array}{llllll}-11 & 4 & 4 & 412 & 384 & 12\end{array}$

$\begin{array}{llllll}-10 & 4 & 4 & 446 & 435 & 13\end{array}$

$\begin{array}{llllll}-9 & 4 & 4 & 959 & 936 & 10\end{array}$

$\begin{array}{llllll}-8 & 4 & 4 & 192 & 111 & 81\end{array}$

$\begin{array}{llllll}-7 & 4 & 4 & 1071 & 1091 & 13\end{array}$

$\begin{array}{llllll}-6 & 4 & 4 & 853 & 852 & 24\end{array}$

$\begin{array}{llllll}-5 & 4 & 4 & 774 & 785 & 23\end{array}$

$\begin{array}{llllll}-4 & 4 & 4 & 225 & 197 & 51\end{array}$

$\begin{array}{llllll}11 & 3 & 6 & 215 & 272 & 22\end{array}$

$\begin{array}{llllll}-11 & 4 & 6 & 209 & 184 & 15\end{array}$

$\begin{array}{llllll}-10 & 4 & 6 & 642 & 634 & 13\end{array}$

$\begin{array}{llllll}-9 & 4 & 6 & 718 & 717 & 12\end{array}$

$\begin{array}{llllll}-8 & 4 & 6 & 402 & 387 & 14\end{array}$

$\begin{array}{llllll}-7 & 4 & 6 & 439 & 439 & 13\end{array}$

$\begin{array}{llllll}-6 & 4 & 6 & 322 & 334 & 24\end{array}$

$\begin{array}{llllll}-5 & 4 & 6 & 166 & 191 & 52\end{array}$

$\begin{array}{llllll}-4 & 4 & 6 & 723 & 722 & 20\end{array}$

$\begin{array}{llllll}-3 & 4 & 6 & 234 & 167 & 53\end{array}$

$\begin{array}{llllll}-2 & 4 & 6 & 208 & 190 & 63\end{array}$

$\begin{array}{llllll}-1 & 4 & 6 & 571 & 602 & 19\end{array}$

$\begin{array}{llllll}0 & 4 & 6 & 824 & 820 & 18\end{array}$

$\begin{array}{llllll}1 & 4 & 6 & 144 & 201 & 79\end{array}$

$\begin{array}{llllll}2 & 4 & 6 & 549 & 534 & 17\end{array}$

$\begin{array}{llllll}3 & 4 & 6 & 268 & 254 & 49\end{array}$

$\begin{array}{llllll}4 & 4 & 6 & 128 & 6 & 127\end{array}$

$\begin{array}{llllll}5 & 4 & 6 & 236 & 160 & 52\end{array}$

$\begin{array}{lllllll}6 & 4 & 6 & 1331 & 1269 & 10\end{array}$

$\begin{array}{llllll}7 & 4 & 6 & 325 & 354 & 41\end{array}$

$\begin{array}{llllll}8 & 4 & 6 & 198 & 212 & 12\end{array}$

$\begin{array}{llllll}9 & 4 & 6 & 193 & 219 & 16\end{array}$

$\begin{array}{llllll}10 & 4 & 6 & 129 & 101 & 34\end{array}$

$\begin{array}{llllll}-10 & 5 & 6 & 73 & 62 & 73\end{array}$

$\begin{array}{llllll}-9 & 5 & 6 & 60 & 91 & 59\end{array}$

$\begin{array}{llllll}-8 & 5 & 6 & 544 & 551 & 12\end{array}$

$\begin{array}{llllll}-7 & 5 & 6 & 268 & 295 & 14\end{array}$

$\begin{array}{lllllll}-6 & 5 & 6 & 361 & 353 & 15\end{array}$

$\begin{array}{llllll}-5 & 5 & 8 & 660 & 697 & 21\end{array}$

$\begin{array}{llllll}-4 & 5 & 6 & 327 & 361 & 25\end{array}$

$\begin{array}{llllll}-3 & 5 & 6 & 265 & 304 & 28\end{array}$

$\begin{array}{llllll}-2 & 5 & 6 & 531 & 467 & 21\end{array}$

$\begin{array}{llllll}-1 & 5 & 6 & 267 & 285 & 43\end{array}$

$\begin{array}{llllll}0 & 5 & 6 & 163 & 109 & 115\end{array}$

$\begin{array}{llllll}1 & 5 & 6 & 87 & 119 & 86\end{array}$

$\begin{array}{llllll}2 & 5 & 6 & 316 & 272 & 30\end{array}$ $\begin{array}{llllll}8 & 7 & 4 & 252 & 265 & 9\end{array}$

$\begin{array}{llllll}7 & 7 & 4 & 171 & 140 & 18\end{array}$

$\begin{array}{llllll}8 & 7 & 4 & 70 & 36 & 70\end{array}$

$\begin{array}{llllll}-7 & 8 & 4 & 165 & 183 & 23\end{array}$

$\begin{array}{llllll}-6 & 8 & 4 & 183 & 206 & 21\end{array}$

$\begin{array}{llllll}-5 & 8 & 4 & 731 & 771 & 13\end{array}$

$\begin{array}{llllll}-4 & 8 & 4 & 668 & 665 & 13\end{array}$

$\begin{array}{lllllll}-3 & 8 & 4 & 683 & 658 & 12\end{array}$

$\begin{array}{llllll}-2 & 8 & 4 & 447 & 465 & 15\end{array}$

$\begin{array}{llllll}-1 & 8 & 4 & 863 & 863 & 10\end{array}$

$\begin{array}{llllll}0 & 8 & 4 & 477 & 469 & 14\end{array}$

$\begin{array}{llllll}1 & 8 & 4 & 170 & 173 & 15\end{array}$

$\begin{array}{llllll}2 & 8 & 4 & 488 & 489 & 14\end{array}$

$\begin{array}{llllll}3 & 8 & 4 & 118 & 60 & 22\end{array}$

$\begin{array}{llllll}4 & 8 & 4 & 383 & 384 & 17\end{array}$

$\begin{array}{llllll}5 & 8 & 4 & 323 & 353 & 9\end{array}$

$\begin{array}{llllll}6 & 8 & 4 & 348 & 342 & 12\end{array}$

$\begin{array}{llllll}-3 & 9 & 4 & 459 & 471 & 15\end{array}$

$\begin{array}{llllll}-2 & 9 & 4 & 159 & 148 & 18\end{array}$

$\begin{array}{llllll}-2 & 0 & 7 & 846 & 872 & 21\end{array}$

$\begin{array}{llllll}0 & 0 & 7 & 171 & 172 & 46\end{array}$

$\begin{array}{llllll}2 & 0 & 7 & 269 & 192 & 38\end{array}$

$\begin{array}{llllll}4 & 0 & 7 & 1702 & 1587 & 17\end{array}$

$\begin{array}{llllll}6 & 0 & 7 & 605 & 556 & 20\end{array}$

$\begin{array}{llllll}8 & 0 & 7 & 249 & 201 & 52\end{array}$

$\begin{array}{llllll}10 & 0 & 7 & 873 & 872 & 12\end{array}$

$\begin{array}{llllll}-12 & 1 & 7 & 340 & 283 & 12\end{array}$

$\begin{array}{llllll}-11 & 1 & 7 & 366 & 347 & 19\end{array}$

$\begin{array}{llllll}-10 & 1 & 7 & 454 & 421 & 13\end{array}$ $\begin{array}{llllll}-9 & 1 & 7 & 220 & 27 & 59\end{array}$ $\begin{array}{llllll}-8 & 1 & 7 & 62 & 154 & 61\end{array}$ $\begin{array}{llllll}-7 & 1 & 7 & 1204 & 1205 & 21\end{array}$ $\begin{array}{llllll}-6 & 1 & 7 & 117 & 94 & 117\end{array}$ $\begin{array}{llllll}-5 & 1 & 7 & 534 & 635 & -26\end{array}$ $\begin{array}{llllll}-4 & 1 & 7 & 127 & 178 & 126\end{array}$ $\begin{array}{llllll}-3 & 1 & 7 & 243 & 260 & 63\end{array}$ $\begin{array}{llllll}-2 & 1 & 7 & 324 & 364 & 37\end{array}$ $\begin{array}{llllll}-1 & 1 & 7 & 217 & 261 & 22\end{array}$ $\begin{array}{llllll}0 & 1 & 7 & 847 & 837 & 17\end{array}$ $\begin{array}{llllll}1 & 1 & 7 & 540 & 510 & 23\end{array}$ $\begin{array}{llllll}2 & 1 & 7 & 190 & 129 & 60\end{array}$ $\begin{array}{llllll}3 & 1 & 7 & 54 & 129 & 53\end{array}$ $\begin{array}{llllll}4 & 1 & 7 & 469 & 462 & 9\end{array}$ $\begin{array}{llllll}5 & 1 & 7 & 340 & 337 & 25\end{array}$ $\begin{array}{llllll}6 & 1 & 7 & 505 & 473 & 36\end{array}$ $\begin{array}{llllll}7 & 1 & 7 & 121 & 138 & 121\end{array}$ $\begin{array}{llllll}8 & 1 & 7 & 330 & 345 & 10\end{array}$ $\begin{array}{llllll}9 & 1 & 7 & 555 & 608 & 9\end{array}$ $\begin{array}{llllll}10 & 1 & 7 & 272 & 276 & 13\end{array}$ $\begin{array}{llllll}11 & 1 & 7 & 118 & 97 & 35\end{array}$ $\begin{array}{llllll}-12 & 2 & 7 & 471 & 424 & 15\end{array}$ $\begin{array}{llllll}-11 & 2 & 7 & 120 & 126 & 25\end{array}$ $\begin{array}{rrrrrr}-10 & 2 & 7 & 104 & 42 & 20\end{array}$ $\begin{array}{llllll}-9 & 2 & 7 & 184 & 152 & 10\end{array}$ $\begin{array}{llllll}-8 & 2 & 7 & 248 & 211 & 33\end{array}$ 
$\begin{array}{llllll}6 & 1 & 5 & 54 & 45 & 53\end{array}$ $\begin{array}{llllll}7 & 1 & 5 & 65 & 117 & 65\end{array}$ $\begin{array}{llllll}8 & 1 & 5 & 636 & 639 & 20\end{array}$ $\begin{array}{llllll}9 & 1 & 5 & 289 & 308 & 18\end{array}$ $\begin{array}{llllll}10 & 1 & 5 & 471 & 515 & 15\end{array}$ $\begin{array}{llllll}11 & 1 & 5 & 771 & 787 & 13\end{array}$ $\begin{array}{llllll}12 & 1 & 5 & 403 & 393 & 12\end{array}$ $\begin{array}{llllll}-12 & 2 & 5 & 344 & 324 & 12\end{array}$ $\begin{array}{llllll}-11 & 2 & 5 & 172 & 160 & 21\end{array}$ $\begin{array}{llllll}-10 & 2 & 5 & 659 & 658 & 12\end{array}$ $\begin{array}{llllll}-9 & 2 & 5 & 534 & 558 & 34\end{array}$ $\begin{array}{lllllll}-8 & 2 & 5 & 1486 & 1525 & 21\end{array}$ $\begin{array}{llllll}-7 & 2 & 5 & 241 & 181 & 47\end{array}$ $\begin{array}{llllll}-6 & 2 & 5 & 203 & 215 & 57\end{array}$ $\begin{array}{llllll}-5 & 2 & 5 & 590 & 605 & 23\end{array}$ $\begin{array}{llllll}-4 & 2 & 5 & 0 & 98 & 1\end{array}$ $\begin{array}{llllll}-3 & 2 & 5 & 481 & 574 & 34\end{array}$ $\begin{array}{llllll}-2 & 2 & 5 & 288 & 267 & 25\end{array}$ $\begin{array}{llllll}-1 & 2 & 5 & 339 & 339 & 40\end{array}$ $\begin{array}{llllll}0 & 2 & 5 & 234 & 220 & 49\end{array}$ $\begin{array}{llllll}1 & 2 & 5 & 0 & 70 & 1\end{array}$ $\begin{array}{llllll}2 & 2 & 5 & 401 & 415 & 36\end{array}$ $\begin{array}{llllll}3 & 2 & 5 & 284 & 256 & 37\end{array}$ $\begin{array}{llllll}4 & 2 & 5 & 0 & 129 & 1\end{array}$ $\begin{array}{llllll}5 & 2 & 5 & 272 & 251 & 52\end{array}$ $\begin{array}{llllll}6 & 2 & 5 & 0 & 14 & 1\end{array}$ $\begin{array}{llllll}7 & 2 & 5 & 787 & 782 & 26\end{array}$ $\begin{array}{llllll}8 & 2 & 5 & 532 & 585 & 23\end{array}$ $\begin{array}{llllll}9 & 2 & 5 & 238 & 222 & 12\end{array}$ $\begin{array}{llllll}10 & 2 & 5 & 82 & 57 & 81\end{array}$ $\begin{array}{llllll}11 & 2 & 5 & 127 & 100 & 33\end{array}$ $\begin{array}{llllll}12 & 2 & 5 & 258 & 292 & 28\end{array}$ $\begin{array}{llllll}-12 & 3 & 5 & 236 & 239 & 14\end{array}$ $\begin{array}{llllll}-11 & 3 & 5 & 90 & 123 & 43\end{array}$ $\begin{array}{llllll}-10 & 3 & 5 & 739 & 744 & 12\end{array}$ $\begin{array}{llllll}-9 & 3 & 5 & 454 & 439 & 14\end{array}$ $\begin{array}{llllll}-8 & 3 & 5 & 914 & 857 & 24\end{array}$ $\begin{array}{llllll}-7 & 3 & 5 & 1221 & 1258 & 22\end{array}$ $\begin{array}{llllll}-6 & 3 & 5 & 521 & 545 & 25\end{array}$ $\begin{array}{llllll}-5 & 3 & 5 & 380 & 432 & 19\end{array}$ $\begin{array}{llllll}-4 & 3 & 5 & 0 & 26 & 1\end{array}$ $\begin{array}{llllll}-3 & 3 & 5 & 211 & 181 & 47\end{array}$ $\begin{array}{llllll}-2 & 3 & 5 & 683 & 745 & 17\end{array}$ $\begin{array}{llllll}-1 & 3 & 5 & 173 & 113 & 60\end{array}$ $\begin{array}{llllll}0 & 3 & 5 & 0 & 3 & 1\end{array}$ $\begin{array}{llllll}1 & 3 & 5 & 453 & 476 & 34\end{array}$ $\begin{array}{llllll}2 & 3 & 5 & 145 & 145 & 61\end{array}$ $\begin{array}{llllll}3 & 3 & 5 & 467 & 494 & 37\end{array}$ $\begin{array}{llllll}4 & 3 & 5 & 0 & 109 & 1\end{array}$ $\begin{array}{llllll}5 & 3 & 5 & 321 & 249 & 40\end{array}$ $\begin{array}{llllll}6 & 3 & 5 & 302 & 253 & 43\end{array}$ $\begin{array}{llllll}7 & 3 & 5 & 594 & 581 & 21\end{array}$ $\begin{array}{llllll}8 & 3 & 5 & 656 & 639 & 43\end{array}$ $\begin{array}{lllllll}9 & 3 & 5 & 558 & 531 & 13\end{array}$ $\begin{array}{llllll}3 & 4 & 7 & 277 & 219 & 38\end{array}$ $\begin{array}{llllll}0 & 5 & 5 & 825 & 841 & 26\end{array}$ $\begin{array}{llllll}1 & 5 & 5 & 146 & 85 & 71\end{array}$ $\begin{array}{llllll}2 & 5 & 5 & 865 & 847 & 23\end{array}$ $\begin{array}{llllll}3 & 5 & 5 & 535 & 515 & 22\end{array}$ $\begin{array}{llllll}4 & 5 & 5 & 159 & 227 & 83\end{array}$ $\begin{array}{llllll}5 & 5 & 5 & 407 & 395 & 23\end{array}$ $\begin{array}{llllll}6 & 5 & 5 & 1003 & 979 & 11\end{array}$ $\begin{array}{llllll}7 & 5 & 5 & 345 & 376 & 15\end{array}$ $\begin{array}{llllll}8 & 5 & 5 & 274 & 304 & 17\end{array}$ $\begin{array}{llllll}9 & 5 & 5 & 100 & 79 & 37\end{array}$ $\begin{array}{llllll}10 & 5 & 5 & 330 & 335 & 14\end{array}$ $\begin{array}{rrrrrr}-10 & 6 & 5 & 0 & 48 & 1\end{array}$ $\begin{array}{llllll}-9 & 6 & 5 & 290 & 341 & 19\end{array}$ $\begin{array}{llllll}-8 & 6 & 5 & 311 & 316 & 12\end{array}$ $\begin{array}{llllll}-7 & 6 & 5 & 207 & 195 & 13\end{array}$ $\begin{array}{llllll}-6 & 6 & 5 & 118 & 100 & 19\end{array}$ $\begin{array}{llllll}-5 & 6 & 5 & 92 & 139 & 50\end{array}$ $\begin{array}{llllll}-4 & 6 & 5 & 991 & 975 & 10\end{array}$ $\begin{array}{llllll}-3 & 6 & 5 & 334 & 326 & 24\end{array}$ $\begin{array}{llllll}-2 & 6 & 5 & 141 & 60 & 140\end{array}$ $\begin{array}{llllll}-1 & 6 & 5 & 655 & 637 & 21\end{array}$ $\begin{array}{llllll}0 & 6 & 5 & 471 & 459 & 34\end{array}$ $\begin{array}{llllll}1 & 6 & 5 & 273 & 273 & 60\end{array}$ $\begin{array}{llllll}2 & 6 & 5 & 294 & 174 & 49\end{array}$ $\begin{array}{llllll}3 & 6 & 5 & 2213 & 2190 & 4\end{array}$ $\begin{array}{llllll}4 & 6 & 5 & 933 & 883 & 10\end{array}$ $\begin{array}{llllll}5 & 8 & 5 & 369 & 377 & 14\end{array}$ $\begin{array}{llllll}6 & 6 & 5 & 437 & 424 & 16\end{array}$ $\begin{array}{llllll}7 & 6 & 5 & 450 & 477 & 14\end{array}$ $\begin{array}{llllll}8 & 6 & 5 & 212 & 224 & 16\end{array}$ $\begin{array}{llllll}9 & 6 & 5 & 79 & 101 & 78\end{array}$ $\begin{array}{lllllll}-8 & 7 & 5 & 300 & 313 & 18\end{array}$ $\begin{array}{llllll}-7 & 7 & 5 & 611 & 642 & 14\end{array}$ $\begin{array}{rrrrrr}-6 & 7 & 5 & 0 & 64 & 1\end{array}$ $\begin{array}{llllll}-5 & 7 & 5 & 306 & 333 & 17\end{array}$ $\begin{array}{llllll}-4 & 7 & 5 & 77 & 81 & 39\end{array}$ $\begin{array}{llllll}-3 & 7 & 5 & 690 & 656 & 11\end{array}$ $\begin{array}{llllll}-2 & 7 & 5 & 89 & 15 & 51\end{array}$ $\begin{array}{lllllll}-1 & 7 & 5 & 315 & 314 & 12\end{array}$ $\begin{array}{llllll}0 & 7 & 5 & 1375 & 1337 & 10\end{array}$ $\begin{array}{llllll}1 & 7 & 5 & 372 & 390 & 14\end{array}$ $\begin{array}{llllll}2 & 7 & 5 & 343 & 352 & 15\end{array}$ $\begin{array}{llllll}3 & 7 & 5 & 497 & 473 & 12\end{array}$ $\begin{array}{llllll}4 & 7 & 5 & 100 & 1 & 25\end{array}$ $\begin{array}{llllll}5 & 7 & 5 & 323 & 333 & 15\end{array}$ $\begin{array}{llllll}6 & 7 & 5 & 215 & 279 & 19\end{array}$ $\begin{array}{llllll}7 & 7 & 5 & 317 & 295 & 12\end{array}$ $\begin{array}{llllll}-6 & 8 & 5 & 137 & 109 & 28\end{array}$ $\begin{array}{llllll}-5 & 8 & 5 & 86 & 46 & 65\end{array}$ $\begin{array}{llllll}-4 & 8 & 5 & 258 & 276 & 14\end{array}$ $\begin{array}{llllll}-3 & 8 & 5 & 247 & 265 & 13\end{array}$ $\begin{array}{llllll}-2 & 8 & 5 & 518 & 557 & 14\end{array}$ $\begin{array}{lllllll}-1 & 8 & 5 & 282 & 385 & -15\end{array}$ $\begin{array}{llllll}0 & 8 & 5 & 160 & 77 & -17\end{array}$ $\begin{array}{llllll}5 & 1 & 8 & 312 & 338 & 20\end{array}$ $\begin{array}{lllllll}5 & 1 & 6 & 141 & 84 & 141\end{array}$ $\begin{array}{llllll}6 & 1 & 6 & 0 & 73 & 1\end{array}$ $\begin{array}{llllll}7 & 1 & 6 & 191 & 96 & 33\end{array}$ $\begin{array}{llllll}8 & 1 & 6 & 117 & 110 & 42\end{array}$ $\begin{array}{lllllll}9 & 1 & 6 & 401 & 417 & 16\end{array}$ $\begin{array}{llllll}10 & 1 & 6 & 524 & 553 & 14\end{array}$ $\begin{array}{llllll}11 & 1 & 6 & 87 & 62 & 66\end{array}$ $\begin{array}{llllll}-12 & 2 & 6 & 194 & 185 & 16\end{array}$ $\begin{array}{llllll}-11 & 2 & 6 & 135 & 159 & 28\end{array}$ $\begin{array}{llllll}-10 & 2 & 6 & 285 & 277 & 12\end{array}$ $\begin{array}{llllll}-9 & 2 & 6 & 1223 & 1186 & 10\end{array}$ $\begin{array}{llllll}-8 & 2 & 6 & 498 & 548 & 35\end{array}$ $\begin{array}{llllll}-7 & 2 & 6 & 968 & 960 & 22\end{array}$ $\begin{array}{llllll}-6 & 2 & 6 & 254 & 178 & 46\end{array}$ $\begin{array}{llllll}-5 & 2 & 6 & 852 & 850 & 22\end{array}$ $\begin{array}{llllll}-4 & 2 & 6 & 145 & 146 & 96\end{array}$ $\begin{array}{llllll}-3 & 2 & 6 & 378 & 389 & 26\end{array}$ $\begin{array}{llllll}-2 & 2 & 6 & 519 & 485 & 25\end{array}$ $\begin{array}{llllll}-1 & 2 & 6 & 974 & 921 & 18\end{array}$ $\begin{array}{llllll}0 & 2 & 6 & 230 & 259 & 30\end{array}$ $\begin{array}{llllll}1 & 2 & 6 & 349 & 389 & 32\end{array}$ $\begin{array}{llllll}2 & 2 & 6 & 387 & 345 & 20\end{array}$ $\begin{array}{llllll}3 & 2 & 6 & 211 & 164 & 58\end{array}$ $\begin{array}{llllll}4 & 2 & 6 & 215 & 87 & 57\end{array}$ $\begin{array}{llllll}5 & 2 & 6 & 449 & 458 & 21\end{array}$ $\begin{array}{llllll}6 & 2 & 6 & 263 & 108 & 47\end{array}$ $\begin{array}{llllll}7 & 2 & 6 & 417 & 423 & 24\end{array}$ $\begin{array}{llllll}8 & 2 & 6 & 142 & 122 & 31\end{array}$ $\begin{array}{lllllll}9 & 2 & 6 & 517 & 534 & 14\end{array}$ $\begin{array}{llllll}10 & 2 & 6 & 90 & 96 & 35\end{array}$ $\begin{array}{llllll}11 & 2 & 6 & 844 & 817 & 12\end{array}$ $\begin{array}{rrrrrr}-12 & 3 & 6 & 98 & 41 & 45\end{array}$ $\begin{array}{llllll}-11 & 3 & 6 & 289 & 278 & 13\end{array}$ $\begin{array}{llllll}-10 & 3 & 6 & 431 & 430 & 15\end{array}$ $\begin{array}{llllll}-9 & 3 & 6 & 764 & 756 & 12\end{array}$ $\begin{array}{llllll}-8 & 3 & 6 & 446 & 461 & 13\end{array}$ $\begin{array}{llllll}-7 & 3 & 6 & 514 & 529 & 31\end{array}$ $\begin{array}{llllll}-6 & 3 & 6 & 602 & 601 & 27\end{array}$ $\begin{array}{llllll}-5 & 3 & 6 & 962 & 1015 & 22\end{array}$ $\begin{array}{llllll}-4 & 3 & 6 & 287 & 340 & 41\end{array}$ $\begin{array}{llllll}-3 & 3 & 6 & 616 & 617 & 17\end{array}$ $\begin{array}{lllllll}-2 & 3 & 8 & 541 & 566 & 18\end{array}$ $\begin{array}{llllll}-1 & 3 & 6 & 21 & 31 & 21\end{array}$ $\begin{array}{llllll}0 & 3 & 6 & 767 & 793 & 28\end{array}$ $\begin{array}{llllll}1 & 3 & 6 & 644 & 628 & 24\end{array}$ $\begin{array}{llllll}2 & 3 & 6 & 0 & 166 & 1\end{array}$ $\begin{array}{llllll}3 & 3 & 6 & 145 & 151 & 145\end{array}$ $\begin{array}{llllll}4 & 3 & 6 & 806 & 803 & 25\end{array}$ $\begin{array}{llllll}5 & 3 & 6 & 168 & 169 & 39\end{array}$ $\begin{array}{llllll}6 & 3 & 6 & 475 & 495 & 18\end{array}$ $\begin{array}{llllll}7 & 3 & 6 & 907 & 873 & 11\end{array}$ $\begin{array}{llllll}8 & 3 & 6 & 271 & 280 & 12\end{array}$ $\begin{array}{llllll}9 & 3 & 6 & 660 & 653 & 12\end{array}$ $\begin{array}{llllll}10 & 3 & 6 & 197 & 189 & 17\end{array}$ $\begin{array}{lllllll}-5 & 6 & 8 & 566 & 588 & 13\end{array}$ $\begin{array}{llllll}3 & 5 & 6 & 343 & 365 & 20\end{array}$ $\begin{array}{llllll}4 & 5 & 6 & 296 & 281 & 30\end{array}$ $\begin{array}{llllll}5 & 5 & 6 & 424 & 421 & 14\end{array}$ $\begin{array}{llllll}6 & 5 & 6 & 150 & 135 & 16\end{array}$ $\begin{array}{llllll}7 & 5 & 6 & 61 & 56 & 61\end{array}$ $\begin{array}{llllll}8 & 5 & 6 & 114 & 68 & 22\end{array}$ $\begin{array}{llllll}9 & 5 & 6 & 316 & 311 & 9\end{array}$ $\begin{array}{llllll}-9 & 6 & 6 & 542 & 519 & 16\end{array}$ $\begin{array}{llllll}-8 & 6 & 6 & 127 & 127 & 25\end{array}$ $\begin{array}{llllll}-7 & 6 & 6 & 180 & 149 & 16\end{array}$ $\begin{array}{llllll}-6 & 6 & 6 & 750 & 744 & 11\end{array}$ $\begin{array}{llllll}-5 & 6 & 6 & 176 & 172 & 22\end{array}$ $\begin{array}{llllll}-4 & 6 & 6 & 140 & 129 & 29\end{array}$ $\begin{array}{llllll}-3 & 6 & 6 & 864 & 839 & 11\end{array}$ $\begin{array}{llllll}-2 & 6 & 8 & 137 & 116 & 137\end{array}$ $\begin{array}{llllll}-1 & 6 & 6 & 1791 & 1791 & 30\end{array}$ $\begin{array}{llllll}0 & 6 & 6 & 1251 & 1272 & 23\end{array}$ $\begin{array}{llllll}1 & 6 & 6 & 283 & 284 & 15\end{array}$ $\begin{array}{llllll}2 & 6 & 6 & 294 & 286 & 13\end{array}$ $\begin{array}{lllllll}3 & 6 & 6 & 1053 & 1075 & 11\end{array}$ $\begin{array}{llllll}4 & 6 & 6 & 318 & 283 & 11\end{array}$ $\begin{array}{llllll}5 & 6 & 6 & 811 & 835 & 12\end{array}$ $\begin{array}{llllll}6 & 6 & 6 & 511 & 482 & 13\end{array}$ $\begin{array}{llllll}7 & 6 & 6 & 756 & 754 & 13\end{array}$ $\begin{array}{lllllll}8 & 6 & 6 & 123 & 154 & 32\end{array}$ $\begin{array}{llllll}-7 & 7 & 6 & 421 & 447 & 19\end{array}$ $\begin{array}{llllll}-6 & 7 & 6 & 427 & 433 & 15\end{array}$ $\begin{array}{llllll}-5 & 7 & 6 & 276 & 281 & 12\end{array}$ $\begin{array}{llllll}-4 & 7 & 6 & 495 & 482 & 14\end{array}$ $\begin{array}{llllll}-3 & 7 & 6 & 661 & 653 & 12\end{array}$ $\begin{array}{llllll}-2 & 7 & 6 & 699 & 690 & 12\end{array}$ $\begin{array}{llllll}-1 & 7 & 6 & 971 & 963 & 11\end{array}$ $\begin{array}{llllll}0 & 7 & 6 & 75 & 93 & 36\end{array}$ $\begin{array}{llllll}1 & 7 & 6 & 807 & 823 & 11\end{array}$ $\begin{array}{llllll}2 & 7 & 6 & 181 & 197 & 19\end{array}$ $\begin{array}{lllllll}3 & 7 & 6 & 1012 & 1007 & 11\end{array}$ $\begin{array}{llllll}4 & 7 & 6 & 467 & 470 & 14\end{array}$ $\begin{array}{llllll}5 & 7 & 6 & 1083 & 1106 \quad 13\end{array}$ $\begin{array}{llllll}6 & 7 & 6 & 348 & 330 & 12\end{array}$ $\begin{array}{llllll}-5 & 8 & 6 & 352 & 380 & 21\end{array}$ $\begin{array}{llllll}-4 & 8 & 6 & 41 & 33 & 40\end{array}$ $\begin{array}{llllll}-3 & 8 & 6 & 410 & 451 & 15\end{array}$ $\begin{array}{llllll}-2 & 8 & 6 & 307 & 294 & 12\end{array}$ $\begin{array}{llllll}-1 & 8 & 6 & 308 & 296 & 12\end{array}$ $\begin{array}{llllll}0 & 8 & 6 & 681 & 677 & 14\end{array}$ $\begin{array}{llllll}1 & 8 & 6 & 567 & 555 & 15\end{array}$ $\begin{array}{llllll}2 & 8 & 8 & 205 & 198 & 16\end{array}$ $\begin{array}{llllll}3 & 8 & 6 & 298 & 372 & 76\end{array}$ $\begin{array}{llllll}4 & 8 & 6 & 229 & 229 & 17\end{array}$ $\begin{array}{llllll}-12 & 0 & 7 & 439 & 391 & 16\end{array}$ $\begin{array}{llllll}-10 & 0 & 7 & 627 & 640 & 12\end{array}$ $\begin{array}{llllll}-8 & 0 & 7 & 77 & 52 & 77\end{array}$ $\begin{array}{llllll}-6 & 0 & 7 & 1317 & 1387 & 17\end{array}$ $\begin{array}{llllll}-4 & 0 & 7 & 92 & 83 & 91\end{array}$ $\begin{array}{llllll}6 & 3 & 9 & 131 & 154 & 19\end{array}$ $\begin{array}{llllll}-7 & 2 & 7 & 237 & 276 & 32\end{array}$ $\begin{array}{llllll}-6 & 2 & 7 & 617 & 579 & 28\end{array}$ $\begin{array}{llllll}-5 & 2 & 7 & 870 & 889 & 22\end{array}$ $\begin{array}{llllll}-4 & 2 & 7 & 485 & 435 & 20\end{array}$ $\begin{array}{llllll}-3 & 2 & 7 & 610 & 650 & 18\end{array}$ $\begin{array}{llllll}-2 & 2 & 7 & 247 & 218 & 34\end{array}$ $\begin{array}{llllll}-1 & 2 & 7 & 312 & 336 & 94\end{array}$ $\begin{array}{llllll}0 & 2 & 7 & 959 & 957 & 20\end{array}$ $\begin{array}{llllll}1 & 2 & 7 & 128 & 86 & 128\end{array}$ $\begin{array}{llllll}2 & 2 & 7 & 61 & 82 & 60\end{array}$ $\begin{array}{llllll}3 & 2 & 7 & 886 & 815 & 23\end{array}$ $\begin{array}{llllll}4 & 2 & 7 & 911 & 855 & 20\end{array}$ $\begin{array}{llllll}5 & 2 & 7 & 213 & 205 & 24\end{array}$ $\begin{array}{llllll}6 & 2 & 7 & 709 & 722 & 27\end{array}$ $\begin{array}{llllll}7 & 2 & 7 & 307 & 291 & 18\end{array}$ $\begin{array}{llllll}8 & 2 & 7 & 600 & 584 & 12\end{array}$ $\begin{array}{llllll}9 & 2 & 7 & 99 & 4 & 24\end{array}$ $\begin{array}{llllll}10 & 2 & 7 & 105 & 130 & 51\end{array}$ $\begin{array}{llllll}-11 & 3 & 7 & 92 & 8 & 56\end{array}$ $\begin{array}{llllll}-10 & 3 & 7 & 451 & 472 & 15\end{array}$ $\begin{array}{llllll}-9 & 3 & 7 & 209 & 166 & 17\end{array}$ $\begin{array}{lllllll}-8 & 3 & 7 & 155 & 145 & 104\end{array}$ $\begin{array}{lllllll}-7 & 3 & 7 & 555 & 544 & 25\end{array}$ $\begin{array}{llllll}-6 & 3 & 7 & 528 & 628 & -35\end{array}$ $\begin{array}{llllll}-5 & 3 & 7 & 290 & 371 & -24\end{array}$ $\begin{array}{llllll}-4 & 3 & 7 & 283 & 296 & 45\end{array}$ $\begin{array}{llllll}-3 & 3 & 7 & 391 & 426 & 20\end{array}$ $\begin{array}{llllll}-2 & 3 & 7 & 981 & 955 & 21\end{array}$ $\begin{array}{llllll}-1 & 3 & 7 & 768 & 739 & 23\end{array}$ $\begin{array}{llllll}0 & 3 & 7 & 917 & 935 & 22\end{array}$ $\begin{array}{llllll}1 & 3 & 7 & 166 & 89 & 77\end{array}$ $\begin{array}{llllll}2 & 3 & 7 & 93 & 85 & 93\end{array}$ $\begin{array}{llllll}3 & 3 & 7 & 521 & 504 & 23\end{array}$ $\begin{array}{llllll}4 & 3 & 7 & 302 & 310 & 25\end{array}$ $\begin{array}{llllll}5 & 3 & 7 & 898 & 879 & 25\end{array}$ $\begin{array}{llllll}6 & 3 & 7 & 573 & 501 & -9\end{array}$ $\begin{array}{llllll}7 & 3 & 7 & 800 & 575 & 13\end{array}$ $\begin{array}{llllll}8 & 3 & 7 & 272 & 269 & 17\end{array}$ $\begin{array}{llllll}9 & 3 & 7 & 232 & 228 & 14\end{array}$ $\begin{array}{llllll}10 & 3 & 7 & 292 & 290 & 14\end{array}$ $\begin{array}{llllll}-11 & 4 & 7 & 151 & 159 & 19\end{array}$ $\begin{array}{llllll}-10 & 4 & 7 & 225 & 167 & 15\end{array}$ $\begin{array}{llllll}-9 & 4 & 7 & 151 & 88 & 23\end{array}$ $\begin{array}{llllll}-8 & 4 & 7 & 123 & 44 & 31\end{array}$ $\begin{array}{llllll}-7 & 4 & 7 & 207 & 89 & 62\end{array}$ $\begin{array}{llllll}-6 & 4 & 7 & 571 & 565 & 49\end{array}$ $\begin{array}{llllll}-5 & 4 & 7 & 1086 & 1065 & 23\end{array}$ $\begin{array}{llllll}-4 & 4 & 7 & 450 & 451 & 23\end{array}$ $\begin{array}{llllll}-3 & 4 & 7 & 70 & 25 & 69\end{array}$ $\begin{array}{llllll}-2 & 4 & 7 & 627 & 634 & 17\end{array}$ $\begin{array}{llllll}-1 & 4 & 7 & 1119 & 1112 & 22\end{array}$ $\begin{array}{llllll}0 & 4 & 7 & 825 & 855 & 32\end{array}$ $\begin{array}{llllll}1 & 4 & 7 & 998 & 988 & 15\end{array}$ $\begin{array}{llllll}2 & 4 & 7 & 989 & 972 & 24\end{array}$ $\begin{array}{llllll}-5 & 2 & 10 & 227 & 256 & 57\end{array}$ 


\begin{tabular}{|c|c|c|c|c|c|c|c|c|c|c|c|c|c|c|c|c|c|c|c|c|c|c|c|c|c|c|c|c|}
\hline 4 & 4 & 7 & 755 & 740 & 27 & 6 & 1 & 8 & 320 & 364 & 37 & -4 & 6 & 8 & 222 & 224 & 13 & 7 & 3 & 9 & 418 & 385 & 17 & -4 & 210 & 622 & 607 & 15 \\
\hline 5 & 4 & 7 & 275 & 337 & 15 & 7 & 1 & 8 & 811 & 814 & 25 & -3 & $\theta$ & 8 & 471 & 474 & 15 & 8 & 3 & 9 & 132 & 91 & 34 & -3 & 210 & 187 & 243 & 82 \\
\hline$\theta$ & 4 & 7 & 0 & 61. & 1 & 8 & 1 & 8 & 723 & 743 & 12 & -2 & 6 & 8 & 431 & 430 & 14 & -9 & 4 & 9 & 289 & 267 & 13 & -2 & 210 & 434 & 391 & 22 \\
\hline 7 & 4 & 71 & 1003 & 957 & 11 & 9 & 1 & 8 & 170 & 143 & 23 & -1 & 6 & 8 & 358 & 328 & 16 & -8 & 4 & 9 & 503 & 468 & 15 & -1 & 210 & 449 & 509 & 29 \\
\hline 8 & 4 & 7 & 246 & 221 & 14 & 10 & 1 & 8 & 0 & 51 & 1 & 0 & 6 & 8 & 511 & 506 & 11 & -7 & 4 & 9 & 149 & 135 & 19 & 0 & 210 & 0 & 48 & 1 \\
\hline 9 & 4 & 7 & 439 & 424 & 12 & -11 & 2 & 8 & 309 & 306 & 13 & 1 & 6 & 8 & 834 & 789 & 11 & -6 & 4 & 9 & 327 & 297 & 18 & 1 & 210 & 1180 & 1157 & 21 \\
\hline 10 & 5 & 7 & 201 & 159 & 16 & -10 & 2 & 8 & 172 & 174 & 18 & 2 & 6 & 8 & 395 & 404 & 10 & -5 & 4 & 9 & 590 & 552 & 13 & 2 & 210 & 21 & 24 & 20 \\
\hline -9 & 5 & 7 & 110 & 89 & 31 & -9 & 2 & 8 & 210 & 230 & 27 & 3 & 6 & 8 & 515 & 504 & 14 & -4 & 4 & 9 & 538 & 584 & 25 & 3 & 210 & 299 & 259 & 40 \\
\hline-8 & 5 & 7 & 312 & 315 & 12 & -8 & 2 & 8 & 414 & 387 & 38 & 4 & 6 & 8 & 122 & 77 & 24 & -3 & 4 & 9 & 740 & 737 & 16 & 4 & 210 & 124 & 65 & 34 \\
\hline-7 & 5 & 7 & 518 & 503 & 13 & -7 & 2 & 8 & 1020 & 1074 & 21 & 5 & 6 & 8 & 570 & 582 & 14 & -2 & 4 & 9 & 811 & 814 & 24 & 5 & 210 & 55 & 92 & 55 \\
\hline-6 & 5 & 7 & 484 & 504 & 12 & -6 & 2 & 8 & 594 & 619 & 30 & 6 & 6 & 8 & 401 & 467 & 19 & -1 & 4 & 9 & 178 & 153 & 84 & 6 & 210 & 309 & 315 & 13 \\
\hline-5 & 5 & 7 & 170 & 74 & 92 & -5 & 2 & 8 & 929 & 1014 & 23 & -6 & 7 & 8 & 428 & 400 & 12 & 0 & 4 & 9 & 410 & 424 & 37 & 7 & 210 & 93 & 22 & 78 \\
\hline-4 & 5 & 7 & 458 & 487 & 21 & -4 & 2 & 8 & 887 & 857 & 22 & -5 & 7 & 8 & 125 & 85 & 24 & 1 & 4 & 9 & 1144 & 1109 & 15 & 8 & 210 & 47 & 67 & 46 \\
\hline-3 & 5 & 7 & 0 & 61 & 1 & -3 & 2 & 8 & 0 & 74 & 1 & -4 & 7 & 8 & 162 & 122 & 28 & 2 & 4 & 9 & 558 & 502 & 41 & -9 & 310 & 198 & 217 & 28 \\
\hline-2 & 5 & 7 & 584 & 586 & 22 & -2 & 2 & 8 & 50 & 63 & 50 & -3 & 7 & 8 & 158 & 158 & 18 & 3 & 4 & 9 & 664 & 632 & 18 & -8 & 310 & 237 & 260 & 14 \\
\hline-1 & 5 & 7 & 714 & 708 & 31 & -1 & 2 & 8 & 295 & 318 & 41 & -2 & 7 & 8 & 272 & 283 & 14 & 4 & 4 & 9 & 0 & 73 & 1 & -7 & 310 & 230 & 209 & 21 \\
\hline 0 & 5 & 7 & 225 & 92 & 71 & 0 & 2 & 8 & 577 & 559 & 19 & -1 & 7 & 8 & 633 & 601 & 14 & 5 & 4 & 9 & 54 & 99 & 53 & -6 & 310 & 333 & 353 & 16 \\
\hline 1 & 5 & 7 & 905 & 857 & 15 & \pm & 2 & 8 & 106 & 204 & 106 & 0 & 7 & 8 & 130 & 103 & 31 & 6 & 4 & 9 & 125 & 139 & 35 & -5 & 310 & 1242 & 1214 & 10 \\
\hline 2 & 5 & 7 & 583 & 554 & 20 & 2 & 2 & 8 & 596 & 588 & 23 & 1 & 7 & 8 & 768 & 753 & 9 & 7 & 4 & 9 & 461 & 450 & 12 & -4 & 310 & 1988 & 1974 & 62 \\
\hline 3 & 5 & 7 & 289 & 283 & 16 & 3 & 2 & 8 & 137 & 13 & 137 & 2 & 7 & 8 & 65 & 59 & 65 & 8 & 4 & 9 & 607 & 610 & 11 & -3 & 310 & 371 & 350 & 23 \\
\hline 4 & 5 & 7 & 128 & 129 & 19 & 4 & 2 & 8 & 410 & 392 & 23 & 3 & 7 & 8 & 501 & 481 & 26 & -8 & 5 & 9 & 321 & 317 & 12 & -2 & 310 & 786 & 782 & 25 \\
\hline 5 & 5 & 7 & 427 & 422 & 13 & 5 & 2 & 8 & 230 & 224 & 28 & 4 & 7 & 8 & 17 & 37 & 16 & -7 & 5 & 9 & 117 & 132 & 39 & -1 & 310 & 0 & 76 & 1 \\
\hline 6 & 5 & 7 & 961 & 916 & 11 & 6 & 2 & 8 & 508 & 515 & 30 & -10 & 0 & 9 & 509 & 483 & 15 & -6 & 5 & 9 & 256 & 283 & 25 & 0 & 310 & 260 & 200 & 53 \\
\hline 7 & 5 & 7 & 315 & 308 & 12 & 7 & 2 & 8 & 962 & 935 & 10 & -8 & 0 & 9 & 844 & 843 & 12 & -5 & 5 & 9 & 409 & 434 & 17 & 1 & 310 & 346 & 397 & 24 \\
\hline 8 & 5 & 7 & 292 & 307 & 14 & 8 & 2 & 8 & 131 & 90 & 29 & -6 & 0 & 9 & 0 & 36 & 1 & -4 & 5 & 9 & 771 & 780 & 12 & 2 & 310 & 232 & 242 & 59 \\
\hline-8 & 6 & 7 & 653 & 632 & 15 & 9 & 2 & 8 & 294 & 317 & 22 & -4 & 0 & 9 & 286 & 268 & 47 & -3 & 5 & 9 & 178 & 160 & 15 & 3 & 310 & 393 & 397 & 14 \\
\hline-7 & 8 & 7 & 350 & 314 & 12 & 10 & 2 & 8 & 285 & 292 & 13 & -2 & 0 & 9 & 177 & 213 & 78 & -2 & 5 & 9 & 172 & 137 & 23 & 4 & 310 & 91 & 56 & 55 \\
\hline-6 & 6 & 7 & 778 & 789 & 12 & -11 & 3 & 8 & 367 & 346 & 12 & 0 & 0 & 9 & 0 & 89 & 1 & -1 & 5 & 9 & 340 & 355 & 17 & 5 & 310 & 233 & 261 & 15 \\
\hline-5 & 6 & 7 & 454 & 448 & 13 & -10 & 3 & 8 & 159 & 126 & 18 & 2 & 0 & 9 & 240 & 248 & 28 & 0 & 5 & 9 & 658 & 679 & 12 & 6 & 310 & 401 & 392 & 12 \\
\hline-4 & 6 & 7 & 444 & 438 & 12 & -9 & 3 & 8 & 110 & 47 & 35 & 4 & 0 & 9 & 2705 & 2611 & 4 & 1 & 5 & 9 & 81 & 12 & 80 & 7 & 310 & 200 & 194 & 31 \\
\hline-3 & 6 & 71 & 1128 & 1103 & 11 & -8 & 3 & 8 & 77 & 63 & 76 & 6 & 0 & 9 & 440 & 423 & 23 & 2 & 5 & 9 & 320 & 350 & 15 & -8 & 410 & 227 & 200 & 15 \\
\hline-2 & 6 & 7 & 579 & 586 & 12 & -7 & 3 & 8 & 325 & 343 & 21 & 8 & 0 & 9 & 970 & 952 & 8 & 3 & 5 & 9 & 252 & 263 & 12 & -7 & 410 & 246 & 224 & 14 \\
\hline-1 & 6 & 7 & 355 & 385 & 15 & -6 & 3 & 8 & 368 & 363 & 33 & -11 & 1 & 9 & 0 & 53 & 1 & 4 & 5 & 9 & 206 & 224 & 20 & -6 & 410 & 215 & 210 & 14 \\
\hline 0 & 6 & 7 & 207 & 174 & 30 & -5 & 3 & 8 & 1413 & 1384 & 16 & -10 & 1 & 9 & 238 & 235 & 19 & 5 & 5 & 9 & 255 & 244 & 14 & -5 & 410 & 802 & 840 & 11 \\
\hline 1. & 6 & 7 & 120 & 88 & 15 & -4 & 3 & 8 & 1450 & 1434 & 10 & -9 & 1 & 9 & 412 & 386 & 13 & 6 & 5 & 9 & 335 & 323 & 13 & -4 & 410 & 207 & 217 & 13 \\
\hline 2 & 6 & 7 & 108 & 71 & 18 & -3 & 3 & 8 & 372 & 343 & 35 & -8 & 1 & 9 & 177 & 170 & 14 & 7 & 5 & 9 & 80 & 6 & 79 & -3 & 410 & 1426 & 1389 & 11 \\
\hline 3 & 6 & 7 & 338 & 317 & 15 & -2 & 3 & 8 & 131 & 131 & 130 & -7 & 1 & 9 & 468 & 492 & 24 & -8 & $B$ & 9 & 237 & 243 & 14 & -2 & 410 & 1015 & 1024 & 10 \\
\hline 4 & 6 & 7 & 374 & 405 & 16 & -1 & 3 & 8 & 97 & 105 & 96 & -6 & 1 & 9 & 230 & 187 & 31 & -5 & 6 & 9 & 126 & 102 & 23 & -1 & 410 & 298 & 328 & 21 \\
\hline 5 & 6 & 7 & 89 & 50 & 54 & 0 & 3 & 8 & 202 & 21.4 & 68 & -5 & 1 & 9 & 736 & 760 & 19 & -4 & 6 & 9 & 205 & 223 & 16 & 0 & 410 & 628 & 601 & 21 \\
\hline 6 & 6 & 7 & 180 & 207 & 18 & 1 & 3 & 8 & 430 & 424 & 23 & -4 & 1 & 9 & 447 & 465 & 29 & -3 & 6 & 9 & 139 & 115 & 27 & 1 & 410 & 64 & 56 & 64 \\
\hline 7 & 6 & 7 & 254 & 272 & 14 & 2 & 3 & 8 & 630 & 608 & 26 & -3 & 1 & 9 & 203 & 187 & 33 & -2 & 6 & 9 & 545 & 538 & 13 & 2 & 410 & 231 & 205 & 13 \\
\hline-7 & 7 & 7 & 373 & 361 & 18 & 3 & 3 & 8 & 481 & 475 & 20 & -2 & 1 & 9 & 0 & 45 & 1 & -1 & 6 & 9 & 539 & 516 & 10 & 3 & 410 & 681 & 681 & 12 \\
\hline-6 & 7 & 7 & 282 & 277 & 25 & 4 & 3 & 8 & 237 & 156 & 38 & -1 & 1 & 9 & 287 & 293 & 44 & 0 & 6 & 9 & 183 & 145 & 16 & 4 & 410 & 204 & 223 & 26 \\
\hline-5 & 7 & 7 & 176 & 142 & 19 & 5 & 3 & 8 & 152 & 119 & 44 & 0 & 1 & 9 & 769 & 737 & 25 & 1 & 6 & 9 & 375 & 376 & 17 & 5 & 410 & 169 & 184 & 26 \\
\hline-4 & 7 & 7 & 490 & 484 & 15 & 6 & 3 & 8 & 139 & 119 & 19 & 1 & 1 & 9 & 0 & 29 & 1 & 2 & 6 & 9 & 349 & 365 & 15 & 6 & 410 & 264 & 378 & -17 \\
\hline-3 & 7 & 7 & 69 & 10 & 69 & 7 & 3 & 8 & 219 & 191 & 13 & 2 & 1 & 9 & 151 & 169 & 126 & 3 & 6 & 9 & 0 & 10 & 1 & 7 & 410 & 0 & 105 & 1 \\
\hline-2 & 7 & 7 & 342 & 335 & 11 & 8 & 3 & 8 & 407 & 452 & 18 & 3 & 1 & 9 & 0 & 9 & 1 & 4 & 6 & 9 & 125 & 127 & 28 & -7 & 510 & 29 & 121 & 28 \\
\hline-1 & 7 & 7 & 170 & 166 & 29 & 9 & 3 & 8 & 243 & 292 & 32 & 4 & 1 & 9 & 82 & 55 & 82 & 5 & 6 & 9 & 1206 & 1222 & 11 & -6 & 510 & 233 & 246 & 15 \\
\hline 0 & 7 & 7 & 236 & 223 & 12 & -10 & 4 & 8 & 290 & 297 & 13 & 5 & 1 & 9 & 526 & 539 & 15 & -4 & 7 & 9 & 323 & 359 & 24 & -5 & 510 & 375 & 375 & 18 \\
\hline 1 & 7 & 7 & 226 & 198 & 14 & -9 & 4 & 8 & 476 & 502 & 17 & 6 & 1 & 9 & 712 & 695 & 24 & -3 & 7 & 9 & 174 & 183 & 19 & -4 & 510 & 154 & 137 & 16 \\
\hline 2 & 7 & 7 & 359 & 377 & 10 & -8 & 4 & 8 & 151 & 135 & 24 & 7 & 1. & 9 & 135 & 145 & 30 & -2 & 7 & 9 & 211 & 189 & 18 & -3 & 510 & 64 & 80 & 63 \\
\hline 3 & 7 & 7 & 787 & 779 & 12 & -7 & 4 & 8 & 268 & 286 & 18 & 8 & 1 & 9 & 625 & 679 & 13 & -1 & 7 & 9 & 253 & 267 & 14 & -2 & 510 & 201 & 248 & 24 \\
\hline 4 & 7 & 7 & 243 & 177 & -15 & -8 & 4 & 8 & 1128 & 1106 & 21 & 9 & 1 & 9 & 564 & 551 & 15 & 0 & 7 & 9 & 301 & 289 & 31 & -1 & 510 & 30 & 51 & 30 \\
\hline 5 & 7 & 7 & 305 & 1329 & 12 & -5 & 4 & 8 & 979 & 1009 & 22 & -10 & 2 & 9 & 0 & 27 & 1 & 1 & 7 & 9 & 88 & 52 & 56 & 0 & 510 & 137 & 110 & 28 \\
\hline & 8 & 7 & & 132 & 52 & -4 & & & 94 & 1217 & 23 & -9 & 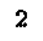 & & 97 & 86 & 41 & 2 & 7 & 8 & 371 & 366 & 10 & 1 & 510 & 103 & 45 & 26 \\
\hline
\end{tabular}


$\begin{array}{llllll}-3 & 8 & 7 & 131 & 100 & 30\end{array}$

$\begin{array}{llllll}-2 & 8 & 7 & 518 & 541 & 12\end{array}$

$\begin{array}{llllll}-1 & 8 & 7 & 145 & 192 & 29\end{array}$

$\begin{array}{llllll}0 & 8 & 7 & 135 & 73 & 27\end{array}$

$\begin{array}{llllll}1 & 8 & 7 & 125 & 103 & 30\end{array}$

$\begin{array}{llllll}2 & 8 & 7 & 774 & 788 & 13\end{array}$

$\begin{array}{llllll}3 & 8 & 7 & 455 & 486 & 15\end{array}$

$\begin{array}{lllllll}-10 & 0 & 8 & 442 & 480 & 14\end{array}$

$\begin{array}{lllllll}-8 & 0 & 8 & 532 & 511 & 29\end{array}$

$\begin{array}{llllll}-6 & 0 & 8 & 932 & 948 & 24\end{array}$

$\begin{array}{llllll}-4 & 0 & 8 & 686 & 682 & 25\end{array}$

$\begin{array}{llllll}-2 & 0 & 8 & 164 & 16 & 76\end{array}$

$\begin{array}{lllllll}0 & 0 & 8 & 250 & 240 & 44\end{array}$

$\begin{array}{llllll}2 & 0 & 8 & 501 & 515 & 26\end{array}$

$\begin{array}{llllll}4 & 0 & 8 & 399 & 384 & 17\end{array}$

$\begin{array}{llllll}6 & 0 & 8 & 1745 & 1676 & -18\end{array}$

$\begin{array}{llllll}8 & 0 & 8 & 819 & 829 & 8\end{array}$

$\begin{array}{llllll}10 & 0 & 8 & 148 & 108 & 29\end{array}$

$\begin{array}{lllllll}-11 & 1 & 8 & 180 & 156 & 22\end{array}$

$\begin{array}{lllllll}-10 & 1 & 8 & 224 & 220 & 13\end{array}$

$\begin{array}{llllll}-9 & 1 & 8 & 126 & 41 & 29\end{array}$

$\begin{array}{llllll}-8 & 1 & 8 & 447 & 470 & 26\end{array}$

$\begin{array}{llllll}-7 & 1 & 8 & 656 & 699 & 29\end{array}$

$\begin{array}{llllll}-6 & 1 & 8 & 849 & 788 & 17\end{array}$

$\begin{array}{llllll}-5 & 1 & 8 & 697 & 666 & 44\end{array}$

$\begin{array}{lllllll}-4 & 1 & 8 & 528 & 558 & 19\end{array}$

$\begin{array}{llllll}-3 & \pm & 8 & 71 & 154 & 70\end{array}$

$\begin{array}{llllll}-2 & 1 & 8 & 776 & 791 & 17\end{array}$

$\begin{array}{llllll}-1 & 1 & 8 & 438 & 443 & 18\end{array}$

$\begin{array}{llllll}0 & 1 & 8 & 336 & 363 & 19\end{array}$

$\begin{array}{llllll}1 & 1 & 8 & 275 & 265 & 48\end{array}$

$\begin{array}{llllll}2 & 1 & 8 & 442 & 416 & 29\end{array}$

$\begin{array}{llllll}3 & 1 & 8 & 867 & 831 & 15\end{array}$

$\begin{array}{llllll}4 & 1 & 8 & 1144 & 1102 & 32\end{array}$

$\begin{array}{llllll}4 & 1 & 11 & 798 & 785 & 11\end{array}$

$\begin{array}{llllll}5 & 1 & 11 & 63 & 104 & 62\end{array}$

$\begin{array}{lllllll}6 & 1 & 11 & 1571 & 1448 & -12\end{array}$

$\begin{array}{llllll}7 & 1 & 11 & 444 & 480 & 20\end{array}$

$\begin{array}{llllll}-8 & 2 & 11 & 370 & 360 & 19\end{array}$

$\begin{array}{llllll}-7 & 2 & 11 & 114 & 1 & 25\end{array}$

$\begin{array}{llllll}-6 & 2 & 11 & 242 & 235 & 10\end{array}$

$\begin{array}{llllll}-5 & 2 & 11 & 231 & 247 & 10\end{array}$

$\begin{array}{llllll}-4 & 2 & 1 & 284 & 304 & 18\end{array}$

$\begin{array}{lllllll}-3 & 2 & 11 & 479 & 488 & 13\end{array}$

$\begin{array}{lllllll}-2 & 2 & 11 & 556 & 524 & 11\end{array}$

$\begin{array}{llllll}-1 & 2 & 11 & 196 & 201 & 26\end{array}$

$\begin{array}{llllll}0 & 2 & 11 & 653 & 701 & 13\end{array}$

$\begin{array}{llllll}1 & 2 & 11 & 153 & 116 & 15\end{array}$

$\begin{array}{llllll}2 & 2 & 11 & 210 & 230 & 14\end{array}$

$\begin{array}{llllll}3 & 2 & 11 & 977 & 958 & 8\end{array}$

$\begin{array}{llllll}4 & 2 & 11 & 139 & 135 & 22\end{array}$

$\begin{array}{llllll}5 & 2 & 11 & 1155 & 1121 \quad 11\end{array}$

$\begin{array}{llllll}6 & 2 & 11 & 184 & 187 & 35\end{array}$

$\begin{array}{lllllll}7 & 2 & 11 & 418 & 460 & 18\end{array}$

$\begin{array}{llllll}-8 & 3 & 11 & 17 & 31 & 16\end{array}$ $\begin{array}{llllll}-3 & 4 & 8 & 233 & 228 & 45\end{array}$

$\begin{array}{llllll}-2 & 4 & 8 & 194 & 281 & 79\end{array}$

$\begin{array}{rrrrrr}-1 & 4 & 8 & 0 & 63 & 1\end{array}$

$\begin{array}{llllll}0 & 4 & 8 & 859 & 835 & 26\end{array}$

$\begin{array}{llllll}1 & 4 & 8 & 412 & 298 & 39\end{array}$

$\begin{array}{llllll}2 & 4 & 8 & 197 & 303 & 99\end{array}$

$\begin{array}{llllll}3 & 4 & 8 & 0 & 12 & 1\end{array}$

$\begin{array}{llllll}4 & 4 & 8 & 392 & 383 & 45\end{array}$

$\begin{array}{llllll}5 & 4 & 8 & 97 & 88 & 45\end{array}$

$\begin{array}{llllll}6 & 4 & 8 & 836 & 852 & 11\end{array}$

$\begin{array}{llllll}7 & 4 & 8 & 759 & 742 & 8\end{array}$

$\begin{array}{llllll}8 & 4 & 8 & 356 & 374 & 16\end{array}$

$\begin{array}{llllll}9 & 4 & 8 & 176 & 217 & 24\end{array}$

$\begin{array}{llllll}-9 & 5 & 8 & 87 & 47 & 65\end{array}$

$\begin{array}{llllll}-8 & 5 & 8 & 556 & 540 & 17\end{array}$

$\begin{array}{llllll}-7 & 5 & 8 & 123 & 15 & -21\end{array}$

$\begin{array}{lllllll}-6 & 5 & 8 & 351 & 338 & 11\end{array}$

$\begin{array}{llllll}-5 & 5 & 8 & 193 & 171 & 14\end{array}$

$\begin{array}{lllllll}-4 & 5 & 8 & 149 & 188 & 148\end{array}$

$\begin{array}{llllll}-3 & 5 & 8 & 172 & 231 & 96\end{array}$

$\begin{array}{llllll}-2 & 5 & 8 & 635 & 586 & 15\end{array}$

$\begin{array}{lllllll}-1 & 5 & 8 & 554 & 564 & 30\end{array}$

$\begin{array}{llllll}0 & 5 & 8 & 223 & 172 & 65\end{array}$

$\begin{array}{llllll}1 & 5 & 8 & 395 & 415 & 19\end{array}$

$\begin{array}{llllll}2 & 5 & 8 & 543 & 519 & 28\end{array}$

$\begin{array}{llllll}3 & 5 & 8 & 251 & 241 & 12\end{array}$

$\begin{array}{llllll}4 & 5 & 8 & 574 & 579 & 13\end{array}$

$\begin{array}{llllll}5 & 5 & 8 & 260 & 267 & 12\end{array}$

$\begin{array}{llllll}6 & 5 & 8 & 377 & 382 & 18\end{array}$

$\begin{array}{llllll}7 & 5 & 8 & 201 & 186 & 16\end{array}$

$\begin{array}{llllll}8 & 5 & 8 & 639 & 635 & 15\end{array}$

$\begin{array}{llllll}-8 & 6 & 8 & 275 & 305 & 14\end{array}$

$\begin{array}{llllll}-7 & 6 & 8 & 408 & 391 & 13\end{array}$

$\begin{array}{llllll}-6 & 6 & 8 & 386 & 389 & 12\end{array}$

$\begin{array}{llllll}0 & 3 & 11 & 809 & 816 & 11\end{array}$

$\begin{array}{lllll}1 & 3 \pm 1 & 22 & 21 & 22\end{array}$

$\begin{array}{llllll}2 & 3 & 11 & 473 & 440 & 14\end{array}$

$\begin{array}{llllll}3 & 3 & 11 & 245 & 253 & 14\end{array}$

$\begin{array}{llllll}4 & 3 & 11 & 641 & 638 & 13\end{array}$

$\begin{array}{llllll}5 & 3 & 11 & 130 & 123 & 36\end{array}$

6 $311 \quad 478 \quad 466 \quad 18$

$\begin{array}{lllllll}-7 & 4 & 11 & 177 & 147 & 22\end{array}$

$\begin{array}{llllll}-6 & 4 & 11 & 451 & 442 & 17\end{array}$

$\begin{array}{llllll}-5 & 4 & 11 & 426 & 398 & 17\end{array}$

$\begin{array}{llllll}-4 & 4 & 11 & 498 & 472 & 14\end{array}$

$\begin{array}{llllll}-3 & 4 & 11 & 383 & 391 & 14\end{array}$

$\begin{array}{llllll}-2 & 4 & 11 & 330 & 309 & 12\end{array}$

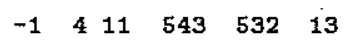

$0 \quad 41130 \quad 3130$

$\begin{array}{llllll}1 & 4 & 11 & 331 & 322 & 16\end{array}$

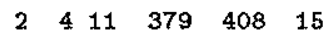

$\begin{array}{llllll}3 & 4 & 11 & 100 & 76 & 56\end{array}$

$\begin{array}{llllll}4 & 4 & 11 & 248 & 353 & -18\end{array}$

$\begin{array}{llllll}5 & 4 & 11 & 318 & 322 & 19\end{array}$

$\begin{array}{llllll}-5 & 5 & 11 & 149 & 113 & 26\end{array}$ $\begin{array}{llllll}-8 & 2 & 9 & 179 & 167 & 14\end{array}$

$\begin{array}{llllll}-7 & 2 & 9 & 159 & 137 & 16\end{array}$

$\begin{array}{llllll}-6 & 2 & 9 & 584 & 578 & 19\end{array}$

$\begin{array}{llllll}-5 & 2 & 9 & 253 & 74 & 56\end{array}$

$\begin{array}{llllll}-4 & 2 & 9 & 338 & 355 & 43\end{array}$

$\begin{array}{lllllll}-3 & 2 & 9 & 781 & 780 & 26\end{array}$

$\begin{array}{llllll}-2 & 2 & 9 & 633 & 637 & 28\end{array}$

$\begin{array}{llllll}-1 & 2 & 9 & 777 & 803 & 25\end{array}$

$\begin{array}{llllll}0 & 2 & 9 & 411 & 418 & 32\end{array}$

$129182 \quad 199$

$\begin{array}{llllll}2 & 2 & 9 & 755 & 724 & 24\end{array}$

$\begin{array}{lllllll}3 & 2 & 9 & 839 & 832 & 24\end{array}$

$\begin{array}{llllll}4 & 2 & 9 & 256 & 164 & 53\end{array}$

$\begin{array}{llllll}5 & 2 & 9 & 92 & 55 & 91\end{array}$

$\begin{array}{llllll}6 & 2 & 9 & 651 & 638 & 13\end{array}$

$\begin{array}{llllll}7 & 2 & 9 & 262 & 265 & 19\end{array}$

$\begin{array}{lllllll}8 & 2 & 9 & 181 & 151 & 18\end{array}$

$\begin{array}{llllll}9 & 2 & 9 & 212 & 208 & 16\end{array}$

$\begin{array}{llllll}-10 & 3 & 9 & 127 & 133 & 32\end{array}$

$\begin{array}{llllll}-9 & 3 & 9 & 81 & 27 & 81\end{array}$

$\begin{array}{llllll}-8 & 3 & 9 & 196 & 181 & 15\end{array}$

$\begin{array}{lllllll}-7 & 3 & 9 & 463 & 476 & 13\end{array}$

$\begin{array}{llllll}-6 & 3 & 9 & 550 & 554 & 29\end{array}$

$\begin{array}{llllll}-5 & 3 & 9 & 193 & 166 & 48\end{array}$

$\begin{array}{llllll}-4 & 3 & 9 & 273 & 5 & -51\end{array}$

$\begin{array}{llllll}-3 & 3 & 9 & 1731 & 1700 & 21\end{array}$

$\begin{array}{llllll}-2 & 3 & 9 & 260 & 240 & 57\end{array}$

$\begin{array}{rrrrrr}-1 & 3 & 9 & 0 & 71 & 1\end{array}$

$\begin{array}{llllll}0 & 3 & 9 & 1201 & 1210 & 22\end{array}$

$\begin{array}{llllll}1 & 3 & 9 & 647 & 622 & 16\end{array}$

$\begin{array}{lllllll}2 & 3 & 9 & 1653 & 1650 & 27\end{array}$

$\begin{array}{llllll}3 & 3 & 9 & 753 & 745 & 37\end{array}$

$\begin{array}{llllll}4 & 3 & 9 & 977 & 925 & 16\end{array}$

$\begin{array}{llllll}5 & 3 & 9 & 0 & 1 & 1\end{array}$

$\begin{array}{llllll}3 & 5 & 11 & 174 & 155 & 14\end{array}$

$\begin{array}{lllll}-8 & 0.12 & 1038 & 992 & 12\end{array}$

$\begin{array}{llllll}-6 & 0 & 12 & 483 & 478 & 16\end{array}$

$\begin{array}{llllll}-4 & 0 & 12 & 179 & 184 & 21\end{array}$

$\begin{array}{llllll}-2 & 0 & 12 & 129 & 62 & 21\end{array}$

$\begin{array}{llllll}0 & 0 & 12 & 128 & 59 & 30\end{array}$

$\begin{array}{llllll}2 & 0 & 12 & 1063 & 1055 & 11\end{array}$

$\begin{array}{llllll}4 & 0 & 12 & 881 & 886 & 13\end{array}$

$\begin{array}{llllll}6 & 0 & 12 & 551 & 580 & 15\end{array}$

$\begin{array}{llllll}-7 & 1 & 12 & 537 & 492 & 16\end{array}$

$\begin{array}{llllll}-6 & 1 & 12 & 78 & 62 & 39\end{array}$

$\begin{array}{llllll}-5 & 1 & 12 & 711 & 715 & 12\end{array}$

$\begin{array}{llllll}-4 & 1 & 12 & 109 & 77 & 26\end{array}$

$\begin{array}{llllll}-3 & 1 & 12 & 982 & 985 & 8\end{array}$

$\begin{array}{lllllll}-2 & 1 & 12 & 734 & 756 & 12\end{array}$

$\begin{array}{lllllll}-1 & 1 & 12 & 510 & 498 & 14\end{array}$

$\begin{array}{lllllll}0 & 1 & 12 & 287 & 327 & 38\end{array}$

$\begin{array}{lllllll}1 & 1 & 12 & 408 & 414 & 14\end{array}$

$\begin{array}{lllllll}2 & 1 & 12 & 137 & 159 & 19\end{array}$

$\begin{array}{lllllll}3 & 1 & 12 & 330 & 330 & 17\end{array}$

$\begin{array}{llllll}4 & 1 & 12 & 848 & 863 & 13\end{array}$ $\begin{array}{llllll}-10 & 0 & 10 & 0 & 42 & 1\end{array}$

$\begin{array}{llllll}-8 & 0 & 10 & 319 & 305 & 15\end{array}$

$\begin{array}{llllll}-6 & 0 & 10 & 1154 & 1158 \quad 21\end{array}$

$\begin{array}{llllll}-4 & 0 & 10 & 0 & 106 & 1\end{array}$

$\begin{array}{llllll}-2 & 0 & 10 & 325 & 329 & 43\end{array}$

$\begin{array}{llllll}0 & 0 & 10 & 846 & 837 & 25\end{array}$

$\begin{array}{lllllll}2 & 0 & 10 & 1267 & 1281 & 22\end{array}$

$\begin{array}{llllll}4 & 0 & 10 & 150 & 37 & 149\end{array}$

$\begin{array}{llllll}6 & 0 & 10 & 978 & 930 & 11\end{array}$

$\begin{array}{llllll}8 & 0 & 10 & 81 & 72 & 41\end{array}$

$\begin{array}{llllll}-10 & 1 & 10 & 118 & 120 & 46\end{array}$

$\begin{array}{llllll}-9 & 1 & 10 & 53 & 47 & 53\end{array}$

$\begin{array}{lllllll}-8 & 1 & 10 & 136 & 146 & 19\end{array}$

$\begin{array}{lllllll}-7 & 1 & 10 & 195 & 189 & 15\end{array}$

$\begin{array}{lllllll}-6 & 1 & 10 & 637 & 650 & 12\end{array}$

$\begin{array}{llllll}-5 & 1 & 10 & 533 & 507 & 28\end{array}$

$\begin{array}{llllll}-4 & 1 & 10 & 151 & 68 & 53\end{array}$

$\begin{array}{lllllll}-3 & 1 & 10 & 441 & 458 & 21\end{array}$

$\begin{array}{llllll}-2 & 1 & 10 & 0 & 64 & 1\end{array}$

$\begin{array}{llllll}-1 & 1 & 10 & 151 & 11 & 77\end{array}$

$\begin{array}{llllll}0 & 1 & 10 & 616 & 619 & 24\end{array}$

$\begin{array}{lllllll}1 & 1 & 10 & 510 & 537 & 17\end{array}$

$\begin{array}{llllll}2 & 1 & 10 & 1071 & 1094 & 33\end{array}$

$\begin{array}{llllll}3 & 1 & 10 & 1010 & 1012 & 20\end{array}$

$\begin{array}{llllll}4 & 1 & 10 & 601 & 624 & 21\end{array}$

$\begin{array}{llllll}5 & 1 & 10 & 228 & 208 & 7\end{array}$

* $110 \quad 990 \quad 956 \quad 11$

$\begin{array}{llllll}7 & 1 & 10 & 50 & 74 & 50\end{array}$

$\begin{array}{llllll}8 & 1 & 10 & 505 & 494 & 17\end{array}$

$\begin{array}{llllll}-10 & 2 & 10 & 154 & 142 & 26\end{array}$

$\begin{array}{lllllll}-9 & 2 & 10 & 113 & 66 & 24\end{array}$

$\begin{array}{lllllll}-8 & 2 & 10 & 140 & 175 & 29\end{array}$

$\begin{array}{llllll}-7 & 2 & 10 & 187 & 159 & 13\end{array}$

$\begin{array}{llllll}-6 & 2 & 10 & 136 & 94 & 28\end{array}$

$\begin{array}{lllllll}-1 & 2 & 12 & 905 & 901 & 10\end{array}$

$\begin{array}{lllllll}0 & 2 & 12 & 586 & 590 & 13\end{array}$

$\begin{array}{llllll}1 & 2 & 12 & 104 & 25 & 27\end{array}$

$\begin{array}{llllll}2 & 2 & 12 & 189 & 160 & 16\end{array}$

$\begin{array}{llllll}3 & 2 & 12 & 192 & 191 & 14\end{array}$

$\begin{array}{lllllll}4 & 2 & 12 & 297 & 349 & 18\end{array}$

$\begin{array}{llllll}5 & 2 & 12 & 192 & 166 & 18\end{array}$

$\begin{array}{llllll}-6 & 3 & 12 & 65 & 5 & 65\end{array}$

$\begin{array}{llllll}-5 & 3 & 12 & 125 & 133 & 35\end{array}$

$\begin{array}{lllllll}-4 & 3 & 12 & 242 & 229 & 14\end{array}$

$\begin{array}{llllll}-3 & 3 & 12 & 967 & 957 & 12\end{array}$

$\begin{array}{lllllll}-2 & 3 & 12 & 131 & 134 & 23\end{array}$

$\begin{array}{lllllll}-1 & 3 & 12 & 888 & 877 & 12\end{array}$

$\begin{array}{llllll}0 & 3 & 12 & 210 & 214 & 16\end{array}$

$\begin{array}{llllll}1 & 3 & 12 & 113 & 72 & 40\end{array}$

$\begin{array}{llllll}2 & 3 & 12 & 584 & 567 & 15\end{array}$

$\begin{array}{llllll}3 & 3 & 12 & 400 & 421 & 18\end{array}$

$\begin{array}{llllll}4 & 3 & 12 & 114 & 63 & 40\end{array}$

$\begin{array}{llllll}-5 & 4 & 12 & 96 & 37 & 47\end{array}$

$\begin{array}{llllll}-4 & 4 & 12 & 305 & 337 & 22\end{array}$

$\begin{array}{llllll}-3 & 4 & 12 & 579 & 579 & 15\end{array}$ $\begin{array}{llllll}2 & 5 & 10 & 130 & 73 & 23\end{array}$

$\begin{array}{llllll}3 & 5 & 10 & 604 & 610 & 15\end{array}$ $\begin{array}{llllll}4 & 5 & 10 & 0 & 14 & 1\end{array}$

$\begin{array}{llllll}5 & 5 & 10 & 159 & 189 & 22\end{array}$

$\begin{array}{lllllll}-5 & 6 & 10 & 320 & 281 & 17\end{array}$

$\begin{array}{llllll}-4 & 6 & 10 & 119 & 31 & 32\end{array}$

$\begin{array}{llllll}-3 & 6 & 10 & 327 & 324 & 14\end{array}$

$\begin{array}{llllll}-2 & 6 & 10 & 533 & 547 & 17\end{array}$

$\begin{array}{llllll}-1 & 6 & 10 & 112 & 114 & 43\end{array}$

$\begin{array}{llllll}0 & 6 & \text { to } & 627 & 641 & 14\end{array}$

$\begin{array}{llllll}1 & 6 & 10 & 583 & 611 & 21\end{array}$

$\begin{array}{llllll}2 & 6 & 10 & 220 & 217 & 16\end{array}$

$\begin{array}{lllllll}3 & 6 & 10 & 503 & 528 & 15\end{array}$

$\begin{array}{llllll}-8 & 0 & 11 & 656 & 621 & 14\end{array}$

$\begin{array}{llllll}-6 & 0 & 11 & 530 & 520 & 13\end{array}$

$\begin{array}{llllll}-4 & 0 & 11 & 280 & 286 & 11\end{array}$

$\begin{array}{lllllll}-2 & 0 & 11 & 2141 & 2192 & 29\end{array}$

$\begin{array}{llllll}0 & 0 & 11 & 428 & 504 & 26\end{array}$

$\begin{array}{lllllll}2 & 0 & 11 & 1117 & 1157 & 11\end{array}$

$\begin{array}{llllll}4 & 0 & 11 & 182 & 187 & 16\end{array}$

$\begin{array}{lllllll}6 & 0 & 11 & 1105 & 1004 & -8\end{array}$

$\begin{array}{llllll}-9 & 1 & 11 & 174 & 148 & 22\end{array}$

$\begin{array}{lllllll}-8 & 1 & 11 & 458 & 440 & 16\end{array}$

$\begin{array}{llllll}-7 & 1 & 11 & 112 & 41 & 24\end{array}$

$\begin{array}{llllll}-6 & 1 & 11 & 376 & 362 & 14\end{array}$

$\begin{array}{llllll}-5 & 1 & 11 & 78 & 22 & 78\end{array}$

$\begin{array}{llllll}-4 & 1 & 11 & 299 & 270 & 14\end{array}$

$\begin{array}{llllll}-3 & 1 & 11 & 179 & 163 & 82\end{array}$

$\begin{array}{llllll}-2 & 1 & 11 & 213 & 208 & 55\end{array}$

$\begin{array}{llllll}-1 & 1 & 11 & 525 & 523 & 22\end{array}$

$\begin{array}{llllll}0 & 1 & 11 & 253 & 177 & 51\end{array}$

$\begin{array}{lllllll}1 & 1 & 11 & 433 & 447 & 24\end{array}$

$\begin{array}{llllll}2 & 1 & 11 & 775 & 788 & 11\end{array}$

$\begin{array}{llllll}3 & 1 & 11 & 18 & 1 & 17\end{array}$

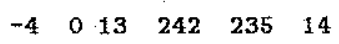

$\begin{array}{lllllll}-2 & 0 & 13 & 716 & 711 & 13\end{array}$

$\begin{array}{llllll}0 & 0 & 13 & 292 & 309 & 13\end{array}$

$\begin{array}{llllll}2 & 0 & 13 & 121 & 236 & 121\end{array}$

$\begin{array}{llllll}-5 & 1 & 13 & 305 & 313 & 13\end{array}$

$\begin{array}{lllllll}-4 & 1 & 13 & 522 & 559 & 13\end{array}$

$\begin{array}{llllll}-3 & 1 & 13 & 0 & 72 & 1\end{array}$

$\begin{array}{llllll}-2 & 1 & 13 & 0 & 65 & 1\end{array}$

$\begin{array}{llllll}-1 & 1 & 13 & 440 & 464 & 16\end{array}$

$\begin{array}{llllll}0 & 1 & 13 & 407 & 412 & 13\end{array}$

$\begin{array}{llllll}1 & 1 & 13 & 644 & 617 & 14\end{array}$

$\begin{array}{llllll}2 & 1 & 13 & 499 & 506 & 17\end{array}$

$\begin{array}{llllll}3 & 1 & 13^{-} & 412 & 399 & 12\end{array}$

$\begin{array}{llllll}-5 & 2 & 13 & 81 & 55 & 81\end{array}$

$\begin{array}{llllll}-4 & 2 & 13 & 156 & 184 & 17\end{array}$

$\begin{array}{llllll}-3 & 2 & 13 & 178 & 206 & 18\end{array}$

$\begin{array}{llllll}-2 & 2 & 13 & 535 & 563 & 16\end{array}$

$\begin{array}{lllllll}-1 & 2 & 13 & 829 & 813 & 13\end{array}$

$\begin{array}{llllll}0 & 2 & 13 & 196 & 199 & 17\end{array}$

$\begin{array}{lllllll}1 & 2 & 13 & 141 & 169 & 31\end{array}$

$\begin{array}{llllll}2 & 2 & 13 & 372 & 349 & 12\end{array}$ 


\begin{tabular}{|c|c|c|c|c|c|c|c|c|c|c|c|c|c|c|c|c|c|c|c|c|c|c|c|c|c|c|c|c|}
\hline-7 & 3 & 11 & 440 & 450 & 17 & -4 & 5 & 11 & 230 & 232 & 16 & 5 & 1 & 12 & 813 & 807 & 14 & -2 & 412 & 1284 & 1236 & 12 & 3 & 21 & 13 & 338 & 313 & 13 \\
\hline-6 & 3 & 11 & 250 & 249 & 12 & -3 & 5 & 11 & 294 & 315 & 29 & -7 & 2 & 12 & 115 & 145 & 37 & -1 & 412 & 283 & 246 & 10 & -3 & 31 & 13 & 358 & 381 & 19 \\
\hline-5 & 3 & 11 & 408 & 418 & 13 & -2 & 5 & 11 & 333 & 325 & 13 & -6 & 2 & 12 & 0 & 38 & 1 & 0 & 412 & 641 & 630 & 14 & -2 & 3 & 131 & 1183 & 1163 & 12 \\
\hline-4 & 3 & 11 & 430 & 421 & 15 & -1 & 5 & 11 & 800 & 771 & 12 & -5 & 2 & 12 & 594 & 624 & 10 & 1 & 4. 12 & 225 & 356 & -24 & -1 & 3 & 13 & 770 & 772 & 13 \\
\hline-3 & 3 & 11 & 763 & 751 & 11 & 0 & 5 & 11 & 301 & 292 & 38 & -4 & 2 & 12 & 296 & 320 & 12 & 2 & 412 & 0 & 30 & 1 & 0 & 3 & 13 & 133 & 71 & 11 \\
\hline-2 & 3 & 11 & 22.3 & 217 & 14 & 1 & 5 & 11 & 282 & 262 & 14 & -3 & 2 & 12 & 82 & 53 & 39 & 3 & 412 & 543 & 599 & 18 & 1 & 31 & 13 & 199 & 185 & \\
\hline-1 & 3 & 11 & 349 & 339 & 16 & 2 & 5 & 11 & 684 & 724 & 14 & -2 & 2 & 12 & 118 & 114 & 33 & -6 & 013 & 298 & 321 & 14 & & & & & & \\
\hline
\end{tabular}

